\title{
Semi-empirical calculation of quenching factors for ions in scintillators
}

\author{
V.I. Tretyak ${ }^{1}$ \\ ${ }^{a}$ Institute for Nuclear Research, MSP 03680 Kyiv, Ukraine \\ ${ }^{\mathrm{b}}$ Department of Physics and Astronomy, Seoul National University, 151-747 \\ Seoul, Republic of Korea
}

\begin{abstract}
Semi-empirical method of calculation of quenching factors for scintillators is described. It is based on classical Birks formula with the total stopping powers for electrons and ions which are calculated with the ESTAR and SRIM codes, respectively. Method has only one fitting parameter (the Birks factor $k B$ ) which can have different values for the same material in different conditions of measurements and data treatment. A hypothesis is used that, once the $k B$ value is obtained by fitting data for particles of one kind and in some energy region (e.g. for a few $\mathrm{MeV} \alpha$ particles from internal contamination of a detector), it can be applied to calculate quenching factors for particles of another kind and for another energies (e.g. for low energy nuclear recoils) if all data are measured in the same experimental conditions and are treated in the same way. Applicability of the method is demonstrated on many examples including materials with different mechanisms of scintillation: organic scintillators (solid $\mathrm{C}_{8} \mathrm{H}_{8}$, and liquid $\mathrm{C}_{16} \mathrm{H}_{18}, \mathrm{C}_{9} \mathrm{H}_{12}$ ); crystal scintillators (pure $\mathrm{CdWO}_{4}, \mathrm{PbWO}_{4}, \mathrm{ZnWO}_{4}, \mathrm{CaWO}_{4}, \mathrm{CeF}_{3}$, and doped $\mathrm{CaF}_{2}(\mathrm{Eu}), \mathrm{CsI}(\mathrm{Tl})$, $\mathrm{CsI}(\mathrm{Na}), \mathrm{NaI}(\mathrm{Tl})$ ); liquid noble gases (LXe). Estimations of quenching factors for nuclear recoils are also given for some scintillators where experimental data are absent $\left(\mathrm{CdWO}_{4}, \mathrm{PbWO}_{4}, \mathrm{CeF}_{3}, \mathrm{Bi}_{4} \mathrm{Ge}_{3} \mathrm{O}_{12}, \mathrm{LiF}, \mathrm{ZnSe}\right)$.
\end{abstract}

Key words: scintillators, quenching factor, $\alpha / \beta$ ratio, dark matter

PACS: 29.40.Mc, 95.35.+d, 32.50.+d

1 Corresponding author. Address: Institute for Nuclear Research, Prospekt Nauki 47, MSP 03680 Kyiv, Ukraine; Telephone: +380 44525 2210; Fax: +380 44525 4463; E-mail address: tretyak@kinr.kiev.ua (Vladimir Tretyak). 


\section{Introduction}

In accordance with our current understanding of astronomical observations, usual matter constitutes only $\simeq 4 \%$ of the Universe; the main components are dark matter $(\simeq 23 \%)$ and dark energy $(\simeq 73 \%)$ [1]. Various extensions of the Standard Model propose many candidates on the role of dark matter (DM) particles [2] which are neutral and only weakly interact with matter (Weakly Interacting Massive Particles, WIMPs). One of the approaches to discover these particles is to detect scattering of WIMPs on atomic nuclei in sensitive detectors placed deep underground and measured in extra low background conditions [3]. Taking into account likely mass range and velocities of WIMPs, energies of nuclear recoils are expected below $\simeq 100 \mathrm{keV}$ with character interaction rates of $1-10^{-6}$ events $\mathrm{kg}^{-1} \mathrm{~d}^{-1}$. Many searches of WIMPs with semiconductor, scintillator and bolometer detectors to-date gave only negative results (see [3] and references therein); instead positive evidence for DM particles (WIMPs are a subclass; other candidates and other kinds of interactions are also available) in the galactic halo has been pointed out by DAMA experiments by exploiting the DM annual modulation signature with $\mathrm{NaI}(\mathrm{Tl})$ scintillators during more than 10 years long measurements [4].

For a long time it is known that amount of light produced in scintillating material by highly ionizing particles is lower than that produced by electrons of the same energy [5]. Thus, in a scintillator calibrated with electron and/or $\gamma$ sources (which is an usual practice), signals from ions will be seen at lower energies (sometimes up to $\simeq 40$ times) than their real values. Evidently knowledge of these transformation coefficients - quenching factors - is extremely important in searches for WIMPs and in predictions where the WIMPs signal should be expected. Many experimental efforts were devoted to measurements, sometimes very sophisticated, of quenching factors at low energies in different detectors (see e.g. recent works [6,7,8,9,10,11,12] and further references).

Quenching factors are also needed in measurements and interpretation of signals from $\alpha$ particles in scintillators. As examples, we can mention here recent experiments on searches (and first observations) of extremely rare $\alpha$ decays $\left(T_{1 / 2}=10^{18}-10^{19} \mathrm{yr}\right):{ }^{180} \mathrm{~W}$ in $\mathrm{CdWO}_{4}$ [13] and $\mathrm{CaWO}_{4}$ [14] crystal scintillators, and ${ }^{151} \mathrm{Eu}$ in $\mathrm{CaF}_{2}(\mathrm{Eu})$ [15].

While few approaches in calculation of quenching factors are known [16, 17, 18, 19], satisfactory theory able to exactly predict (and very often even to describe already measured) quenching factors for all detectors and particles still is absent. For example, in the Lindhard's approach [18 $]_{2}^{2}$ it is possible to calculate quenching of ions with atomic number $Z$ in scintillator only with the same $Z$

$\overline{2 \text { Good }}$ description is given in more accessible source [20]. 
number; in addition, this theory predicts decrease of quenching factors at low energies, very often in contradiction with experimental data. Hitachi's model [19] gives better description and for wider data range, however it is not easy to reproduce these calculations independently.

Below we describe rather simple method of calculation of quenching factors for different ions (from protons to heavy recoils), based on semi-empirical approach of Birks [16] and using available in Internet software for calculation of stopping powers for electrons and ions (ESTAR [21] and SRIM [22] codes, respectively). It employs only one parameter ( $k B$ Birks factor) which could be found by fitting experimental data measured for particles of one kind in some energy region (e.g. for $\alpha$ particles from external sources or internal contamination of a detector by $\mathrm{U} / \mathrm{Th}$ chains, ${ }^{147} \mathrm{Sm},{ }^{190} \mathrm{Pt}$, etc.) but afterwards can be used to calculate quenching factors for other particles and in other energy regions (e.g. for nuclear recoils at low energies). Summary of the method is given in section 2. Calculations with this method are demonstrated in section 3 for number of scintillators: organic scintillators (solid $\mathrm{C}_{8} \mathrm{H}_{8}$, and liquid $\mathrm{C}_{16} \mathrm{H}_{18}, \mathrm{C}_{9} \mathrm{H}_{12}$ ); crystal scintillators (pure $\mathrm{CdWO}_{4}, \mathrm{PbWO}_{4}, \mathrm{ZnWO}_{4}$, $\mathrm{CaWO}_{4}, \mathrm{CeF}_{3}$, and doped $\left.\mathrm{CaF}_{2}(\mathrm{Eu}), \mathrm{CsI}(\mathrm{Tl}), \mathrm{CsI}(\mathrm{Na}), \mathrm{NaI}(\mathrm{Tl})\right)$; liquid noble gases (LXe). Estimations of quenching factors for nuclear recoils are also given for some scintillators where experimental data are absent $\left(\mathrm{CdWO}_{4}, \mathrm{PbWO}_{4}\right.$, $\left.\mathrm{CeF}_{3}, \mathrm{Bi}_{4} \mathrm{Ge}_{3} \mathrm{O}_{12}, \mathrm{LiF}, \mathrm{ZnSe}\right)$. Section 4 gives conclusions.

\section{Outlines of the method}

In calculation of quenching factors, we follow Birks approach in description of quenching of the light yield for highly ionizing particles [5,16]. Light yield of scintillating material depends not only on energy of particle $E$ but also on how big is its stopping power $d E / d r$ in the material. Fig. 1 gives example of stopping powers for electrons (calculated with the ESTAR software [21]), and for protons, alpha particles, $\mathrm{O}, \mathrm{Ca}$ and $\mathrm{W}$ ions (calculated with the SRIM code [22]) in the $\mathrm{CaWO}_{4}$ material.

In case when created in a scintillator excitation centers are spaced at large distances and interactions between them can be neglected, what is realized for particles with low stopping power (fast electrons, energies above $E \simeq 100$ $\mathrm{keV}$ ), scintillation yield $d L$ is proportional to released energy $d E: d L=S d E$ (where $S$ is the absolute scintillation factor), or in differential form

$$
\frac{d L}{d r}=S \frac{d E}{d r}
$$

To account for suppression of the light yield for highly ionizing particles (pro- 

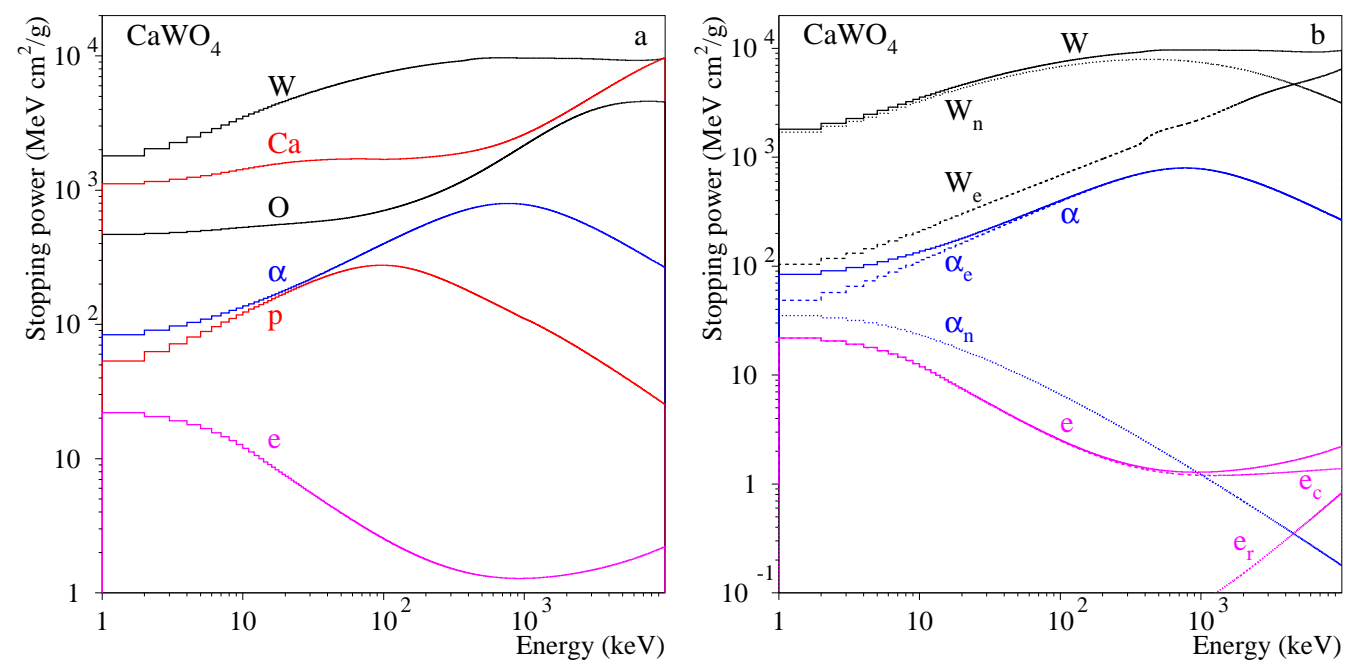

Fig. 1. (Color online) Stopping powers for electrons, protons, $\alpha$ particles, O, Ca and W ions in $\mathrm{CaWO}_{4}$. In (a) only total $d E / d r$ are given; in (b) also nuclear (dotted line) and electronic (dashed line) parts of $d E / d r$ are drawn for $\alpha$ particles and $\mathrm{W}$ ions; for electrons, collision and radiation parts are shown.

tons, $\alpha$ particles and nuclear recoils; hereafter all of them will be named "ions"), Birks proposed semi-empirical formula [5, 16]:

$$
\frac{d L}{d r}=\frac{S \frac{d E}{d r}}{1+k B \frac{d E}{d r}}
$$

where $B d E / d r$ is density of excitation centers along the track, and $k$ is a quenching factor; $k B$ is usually treated as a single parameter (Birks factor).

Equation (2) gives the following approximations for light yields for particles with low (fast electrons) and high (ions) stopping power:

$$
L_{e}(E)=S E, \quad L_{i}(E)=\frac{S r}{k B}
$$

but in general light yield is:

$$
L(E)=\int_{0}^{E} d L=\int_{0}^{E} \frac{S d E}{1+k B \frac{d E}{d r}} .
$$

Quenching factor for ions 3 is a ratio of light yield of ions to that of electrons

$\overline{3}$ Quenching factor for $\alpha$ particles is often named " $\alpha / \beta$ " ratio. 
of the same energy:

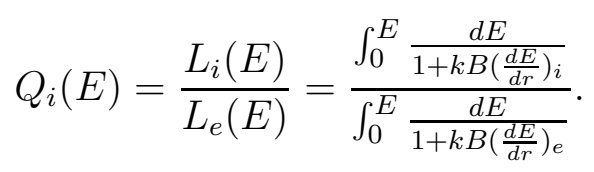

The $S$ factor disappeared in the ratid 4 , and $Q_{i}(E)$ depends only on single parameter $k B$.

Instead of quenching factor, sometimes a relative light yield, ratio of $L_{i}$ to energy $E$, normalized to that for electron $L_{e}$ at some energy $E_{0}$, is used:

$$
R_{i}(E)=\frac{L_{i}(E) / E}{L_{e}\left(E_{0}\right) / E_{0}}
$$

Relation between $Q_{i}(E)$ and $R_{i}(E)$ is evident:

$$
R_{i}(E)=\frac{L_{i}(E)}{L_{e}(E)} \frac{L_{e}(E) / E}{L_{e}\left(E_{0}\right) / E_{0}}=Q_{i}(E) \frac{L_{e}(E) / E}{L_{e}\left(E_{0}\right) / E_{0}} .
$$

Thus, $R_{i}(E)$ is close to $Q_{i}(E)$ if $E$ and $E_{0}$ are in energy region where electron light yield $L_{e}$ is proportional to energy.

Taking into account that approximately (see Eq. (3)) $d L_{e} / d E=S$ and $d L_{i} / d E=$ $\frac{S}{k B} \frac{1}{(d E / d r)_{i}}$, we can obtain the following approximation for quenching factor:

$$
Q_{i}(E)=\frac{L_{i}(E)}{L_{e}(E)}=\frac{L_{i}(E) / E}{L_{e}(E) / E} \simeq \frac{d L_{i} / d E}{d L_{e} / d E} \simeq \frac{1}{k B(d E / d r)_{i}} .
$$

While this expression 5 is approximate, it gives the following important features of quenching factor:

(1) Quenching factor depends on energy. This is not so trivial feature because in many papers on $Q_{i}$ measurements it was supposed that $Q_{i}$ is constant;

(2) $Q_{i}$ is minimal when $(d E / d r)_{i}$ is maximal;

(3) $Q_{i}$ increases at low energies; this is a consequence of decrease of $(d E / d r)_{i}$, see Fig. 1.

$\overline{4}$ Thus, the $S$ factor is supposed independent on energy and equal for electrons and ions; in the following we will suppose that the $k B$ factor is also independent on energy.

5 In [5] it was erroneously written as $Q_{i}=(d E / d r)_{i} / k B$, see Eq. (6.5). 
In the following, we will use Eq. (5) to calculate quenching factors for different particles and different scintillators. Stopping powers for ions will be calculated with the SRIM code [22]. It should be noted that it is possible to calculate stopping powers for $\alpha$ particles and protons also with the ASTAR and PSTAR codes of the STAR package [21, respectively, but list of materials available is restricted. There is also difference in $d E / d r$ calculated with the SRIM and ASTAR \& PSTAR for $\alpha$ particles and protons which results also in difference in calculated quenching factors; some examples are given later. The SRIM code does not allow to calculate stopping powers for electrons, and for this the ESTAR code [21] will be used. Contrary to other approaches, we will use total stopping powers instead of using only electronic part of $d E / d r$. Currently we accept this as a hypothesis and will show in future that it works well (in particular, see Fig. 9 later).

Before to calculate quenching factors and compare them with experimental values measured in different works, the following general note should be made. Quenching factors could depend on many conditions of experimental measurements:

(1) If scintillator is not pure but doped with some material which enhances its scintillating characteristics (e.g. $\mathrm{Tl}$ in $\mathrm{NaI}(\mathrm{Tl})$ or $\mathrm{CsI}(\mathrm{Tl})$ ), $Q_{i}$ depends on kind and amount of dopant. For example, for $\mathrm{PbWO}_{4}$ detectors and external $\alpha$ particles of $5.25 \mathrm{MeV} Q_{i}$ were measured as $Q_{i}=0.19-0.32$ with different dopants and their different amounts [23]. And even if some material is considered as a "pure" scintillator, usually it also contains impurities and defects which could affect $Q_{i}$.

(2) Scintillation dynamics and light output depend on temperature. This is also well known experimental fact; f.e. we can quote Ref. [24] where average decay time of $\mathrm{CaMoO}_{4}$ scintillator was measured as $\simeq 17 \mu \mathrm{s}$ at $+20^{\circ} \mathrm{C}$ but as $\simeq$ $350 \mu \mathrm{s}$ at $-140^{\circ} \mathrm{C}$. Usually scintillation has few components with amplitudes different for different particles. Temperature dependence of these amplitudes could lead to change in quenching factors; for example, for $\alpha$ particle with $E_{\alpha}=2.14 \mathrm{MeV}$ emitted in $\alpha$ decay of ${ }^{152} \mathrm{Gd}$ inside $\mathrm{Gd}_{2} \mathrm{SiO}_{5}(\mathrm{Ce})$ detector, the $\alpha / \beta$ ratio changed from 0.168 at $-20^{\circ} \mathrm{C}$ to 0.178 at $+20^{\circ} \mathrm{C}$ [25]. In Ref. [26], change in temperature from $+20^{\circ} \mathrm{C}$ to $-20^{\circ} \mathrm{C}$ resulted in increase of the $\alpha / \beta$ ratio on $7 \%$ in $\mathrm{NaI}(\mathrm{Tl}), 35 \%$ in $\mathrm{CsI}(\mathrm{Tl})$ and $25 \%$ in $\mathrm{CsI}(\mathrm{Na})$; change from $+20^{\circ} \mathrm{C}$ to $+80^{\circ} \mathrm{C}$ decreased $\alpha / \beta$ ratio on $3 \%, 15 \%$ and $30 \%$ in these crystals, respectively.

(3) Such a technical parameter as time $\Delta t$ during which scintillation signal is collected by a data acquisition system, is in fact very important and could drastically change $Q_{i}$ values. This is because the amplitudes of different components of scintillation signal are different for different particles; thus different parts of a signal will be collected during $\Delta t$. This is illustrated by Fig. 2 where 
relative light output for $\mathrm{CsI}(\mathrm{Tl})$ and external $\alpha$ particles with energies between $\simeq 0$ and $10 \mathrm{MeV}$ were measured with $\Delta t=1 \mu \mathrm{s}$ and $7 \mu \mathrm{s}$ [27]. Attempts to fit these data also are shown. Descriptions of the data with Eq. (6) (which is better for $\Delta t=7 \mu \mathrm{s}$ ) lead to different values of the $k B$ parameter: $k B=1.1 \times 10^{-3}$ $\mathrm{g} \mathrm{MeV}^{-1} \mathrm{~cm}^{-2}$ for $\Delta t=1 \mu \mathrm{s}$, and $k B=2.3 \times 10^{-3} \mathrm{~g} \mathrm{MeV}^{-1} \mathrm{~cm}^{-2}$ for $\Delta t=7$ $\mu \mathrm{s}$. This gives very important conclusion: the Birks factor $k B$, which very often is named "Birks constant", in fact is not a fundamental constant for a given material but could have different values at different experimental conditions (including time of a signal collection $\Delta t$ ).

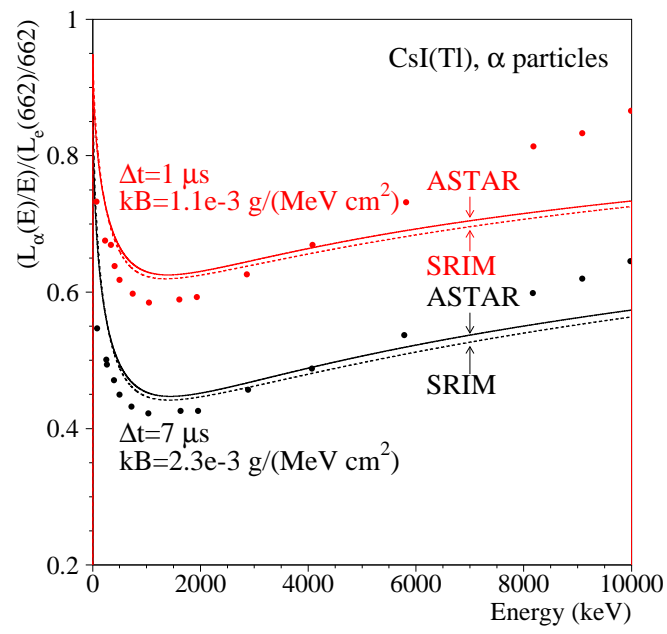

Fig. 2. (Color online) Relative light output (normalized to that for electrons of 662 $\mathrm{keV}$ ) for $\operatorname{CsI}(\mathrm{Tl})$ and $\alpha$ particles [27. Fits of the data with Eq. (6) are shown as continuous curves calculated with the ESTAR (for electrons), and ASTAR or SRIM (for $\alpha$ 's) codes.

If signals are not collected during a proper time, it is possible to obtain wrong conclusions on $Q_{i}$ values. For example, the light output for protons of $662 \mathrm{keV}$ in $\mathrm{CsI}(\mathrm{Tl})$ measured during $\Delta t=1 \mu \mathrm{s}$ in [27] is higher than that for $\gamma$ quanta of the same energy because scintillation signal for $p$ is faster. Thus, instead of expected quenching $\left(Q_{i}<1\right)$ we, on contrary, obtain enhancement $\left(Q_{i}>1\right)$. However, with $\gamma$ and $p$ signals collected during longer time of $7 \mu \mathrm{s}$, we have the usual situation when $Q_{i}<1[27$.

(4) Sometimes quenching factors are derived from measurements with nonmonoenergetic neutron sources like Am-B $\AA^{6}$. After collision with neutron, nuclear recoil with mass $M_{r}$ has energy determined by initial energy of neutron $E_{n}$ and neutron scattering angle $\theta$ :

$$
E_{r}=\frac{2 E_{n}}{(1+\mu)^{2}}\left(\mu+\sin ^{2} \theta-\cos \theta \sqrt{\mu^{2}-\sin ^{2} \theta}\right) \simeq \frac{2 E_{n}}{\mu}(1-\cos \theta),
$$

6 Spectrum of neutrons from Am-Be (Am-B) source has complex structure with energies up to $\simeq 11 \mathrm{MeV}(\simeq 5.5 \mathrm{MeV})[28]$. 
where $\mu=M_{r} / m_{n}, m_{n}$ is the neutron mass, and the last approximation is valid for heavy nuclei $(\mu \gg 1)$. Energy dependence of quenching factor can be found only if the initial neutron energy and scattering angle are known; measurements with non-monoenergetic neutron sources give only some average $Q_{i}$ value which, nevertheless, could be useful estimate of $Q_{i}$ for energy range $\simeq 0-4 E_{n} / A$, where $A$ is mass number of nuclear recoil, if more detailed data are absent (this effective energy range is also related with dependence of the cross section on angle of scattering).

(5) Presence of electric field in case of liquid noble gases could distort initial $Q_{i}$ values (obtained without electric field). Other phenomena like channeling effect in crystals (see f.e. [29]), dependence of $Q_{i}$ on direction of particle's movement relatively to crystal axes (as f.e. in $\mathrm{CdWO}_{4}$ [13]) or diffusion and movement of molecules in liquids [30] also effect quenching factors.

In accordance with the above mentioned, we will not expect that the Birks factor $k B$ for a given material will have the same value in different measurements. However, we will expect that if conditions of measurements and data treatment are fixed, $k B$ will be the same for all particles. Such a hypothesis was discussed already in [5], and it was supported by some experimental data. Below we show that it gives reliable results for a range of energies of interest here (low energy ions and $\alpha$ particles with energies up to $\simeq 10 \mathrm{MeV}$ ).

\section{Calculation of quenching factors}

Results of calculation of quenching factors with Eq. (5), or relative light outputs with Eq. (6) are presented below for a number of organic, crystal and liquid noble gases scintillators. To compare calculations with experimental results, among big number of experimental papers we mainly chose more recent articles where data were obtained with better techniques (monoenergetic neutron beam instead of Am-Be source, for example) or/and in wider energy range.

\subsection{Organic scintillators}

\subsubsection{Polystyrene $\left(\mathrm{C}_{8} \mathrm{H}_{8}\right)$}

Relative light output $L_{\alpha}(E) / E$ for $\alpha$ particles with energies of $2-9 \mathrm{MeV}$ (normalized to that for electrons of $976 \mathrm{keV}$ ) for polystyrene scintillator

$\overline{7}$ Difference between the relative light output and quenching factor for presented energies is small. 
(chemical formula $\mathrm{C}_{8} \mathrm{H}_{8}$, density $\rho=1.06 \mathrm{~g} \mathrm{~cm}^{-3}$ ) was measured recently in Ref. [31] using external $\alpha$ particles from ${ }^{241} \mathrm{Am}$ source with a set of thin mylar absorbers, and internal $\alpha$ particles from contamination of the scintillator by $\mathrm{U} / \mathrm{Th}$ chains. Fit of these experimental data by calculations with Eq. (6) and with the Birks factor $k B=9.0 \times 10^{-3} \mathrm{~g} \mathrm{MeV}^{-1} \mathrm{~cm}^{-2}$ is presented in Fig. 3a. Fitting curves were obtained with stopping powers calculated with the SRIM or ASTAR codes for $\alpha$ particles, and ESTAR code for electrons. Difference between the ASTAR and SRIM calculations is not very big, however $\chi^{2} /$ n.d.f. value 8 for the ASTAR (0.50) is better than that for the $\operatorname{SRIM}(0.58)$.

\subsection{2 $P X E\left(\mathrm{C}_{16} \mathrm{H}_{18}\right)$}

In Ref. [32], quenching factors for $\alpha$ particles were investigated for liquid scintillator: phenyl-o-xylylethane (1,2-dimethyl-4-(1-phenylethyl)-benzene, PXE, chemical formula $\mathrm{C}_{16} \mathrm{H}_{18}$, density $\rho=0.988 \mathrm{~g} \mathrm{~cm}^{-3}$ ) doped with para-Terphenyl (1,4-diphenylbenzene, $\mathrm{p}$-Tp) at $2.0 \mathrm{~g} / \mathrm{l}$ and bis-MSB (1,4-bis(2-methylstyryl)benzene) at $20 \mathrm{mg} / \mathrm{l}$. Alpha particles belong to internal contamination of scintillator by daughters from ${ }^{238} \mathrm{U}$ chain $\left({ }^{222} \mathrm{Rn},{ }^{218} \mathrm{Po},{ }^{214} \mathrm{Po},{ }^{210} \mathrm{Po}\right) ; E_{\alpha}=$ $5.3-7.7 \mathrm{MeV}$.

In fact, in case of $\alpha$ decay of nucleus inside a scintillator, released light output has two components: from $\alpha$ particle and from nuclear recoil. However, corrections for the light output from heavy nuclear recoils are small: for example, for $\alpha$ decay of ${ }^{210} \mathrm{Po}\left(Q_{\alpha}=5407 \mathrm{keV}\right)$ corresponding energies are $E_{\alpha}=5304$ $\mathrm{keV}, E_{r}=103 \mathrm{keV}$ and with the value of $k B=6.8 \times 10^{-3} \mathrm{~g} \mathrm{MeV}^{-1} \mathrm{~cm}^{-2}$ (see Fig. 3b) the light output from ${ }^{206} \mathrm{~Pb}$ recoil is only $2.1 \%$ of that from the $\alpha$ particle. We will neglect these corrections in the following. Quenching factors calculated for $\mathrm{C}_{16} \mathrm{H}_{18}$ (PXE) with $k B=6.8 \times 10^{-3} \mathrm{~g} \mathrm{MeV}^{-1} \mathrm{~cm}^{-2}$ are shown in Fig. 3b in comparison with the experimental data 32$]\left(\chi^{2} /\right.$ n.d.f. $\left.=0.08\right)$.

\subsubsection{Pseudocumene $\left(\mathrm{C}_{9} \mathrm{H}_{12}\right)$}

(1) In Ref. [32], quenching factors for $\alpha$ particles were studied as above also for another liquid scintillator: pseudocumene (1,2,4-trimethylbenzene, $\mathrm{PC}, \mathrm{C}_{9} \mathrm{H}_{12}$, $\rho=0.876 \mathrm{~g} \mathrm{~cm}^{-3}$ ) doped with 2,5-diphenyloxazole (PPO) at a concentration of $1.5 \mathrm{~g} / \mathrm{l}$. Fit of these data $\left(\chi^{2} /\right.$ n.d.f. $\left.=0.12\right)$ is shown in Fig. $3 \mathrm{c}$ with $k B=$ $9.4 \times 10^{-3} \mathrm{~g} \mathrm{MeV}^{-1} \mathrm{~cm}^{-2}$.

(2) Relative light outputs $L_{i}(E) / E$ for protons and C ions in pseudocumene (BC505 liquid scintillator) were measured in [33] in range of energies of 29-943 $\mathrm{keV}$ (protons) and $46-500 \mathrm{keV}$ (C ions). They were normalized to relative

8 Values of $\chi^{2} /$ n.d.f. are calculated everywhere without taking into account uncertainties in energy. 

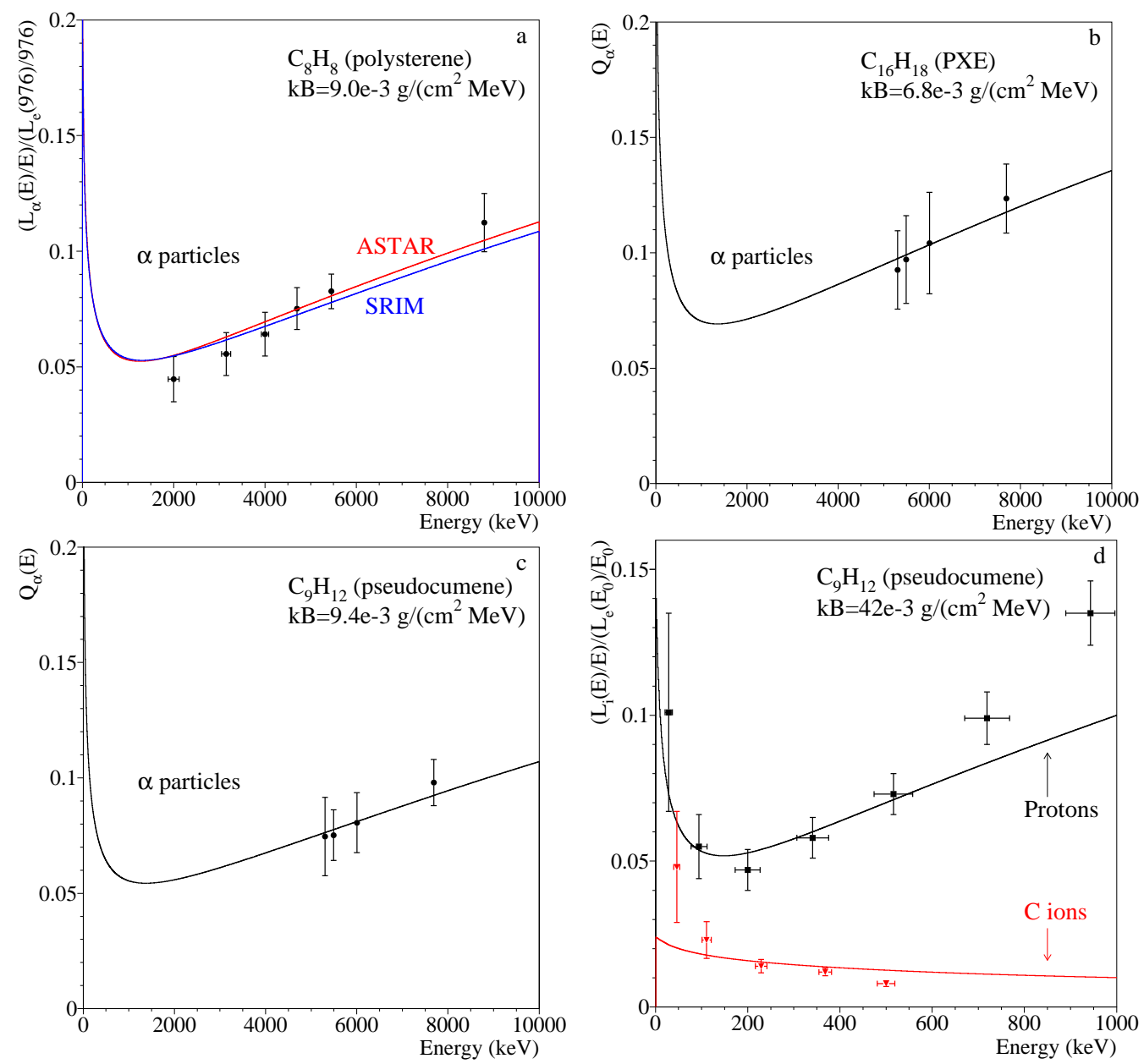

Fig. 3. (Color online) Quenching factors or relative light outputs for $\alpha$ particles and ions in organic scintillators: experimental data and their fit with Eq. (5) or (6). (a) $\mathrm{C}_{8} \mathrm{H}_{8}$ scintillator (polystyrene) [31], $k B=9.0 \times 10^{-3} \mathrm{~g} \mathrm{MeV}^{-1} \mathrm{~cm}^{-2}$; (b) $\mathrm{C}_{16} \mathrm{H}_{18}$ liquid scintillator (PXE) [32, $k B=6.8 \times 10^{-3} \mathrm{~g} \mathrm{MeV}^{-1} \mathrm{~cm}^{-2}$; (c) $\mathrm{C}_{9} \mathrm{H}_{12}$ liquid scintillator (pseudocumene) [32], $k B=9.4 \times 10^{-3} \mathrm{~g} \mathrm{MeV}^{-1} \mathrm{~cm}^{-2}$; (d) $\mathrm{C}_{9} \mathrm{H}_{12}$ [33], $k B=35 \times 10^{-3} \mathrm{~g} \mathrm{MeV}^{-1} \mathrm{~cm}^{-2} ; E_{0}=22 \mathrm{keV}$ for protons and $32 \mathrm{keV}$ for C ions. In part (a), results with stopping powers calculated with the ASTAR and SRIM codes for $\alpha$ particles are shown.

light output for electrons at $22 \mathrm{keV}$ (for $p$ ) and $32 \mathrm{keV}$ (for $\mathrm{C}$ ) [33]. The Birks factor $k B=42 \times 10^{-3} \mathrm{~g} \mathrm{MeV}^{-1} \mathrm{~cm}^{-2}$ was found by fitting the experimental data for protons by Eq. (6) (see Fig. 3d); $\chi^{2} /$ n.d.f. value is 2.8 , with main contribution due to the last experimental point. After this, the curve for $\mathrm{C}$ ions was calculated with this $k B$ value. Comparison of the curve with the $\mathrm{C}$ data gives $\chi^{2} /$ n.d.f. $=5.3$. While this value is high, nevertheless calculations are in a proper agreement with the measured data, as one can see in Fig. $3 \mathrm{~d}$.

The $k B$ values for pseudocumene $\mathrm{C}_{9} \mathrm{H}_{12}$ obtained by fitting data of [32] and [33], respectively (Fig. 3c and 3d), are quite different. However, this is not a surprise taking into account different conditions of measurements and probably 
dopants used. Nevertheless, the same $k B$ value fixed in one experiment [33] allowed to describe both data sets: for protons and $\mathrm{C}$ ions.

\subsection{Crystal scintillators}

\subsection{1 $\mathrm{CdWO}_{4}$}

(1) Quenching factors for $\alpha$ particles in $\mathrm{CdWO}_{4}$ crystal scintillator (density $\rho=7.9 \mathrm{~g} \mathrm{~cm}^{-3}$ ) were measured in experimental searches for rare $\alpha$ decay of ${ }^{180} \mathrm{~W}\left(Q_{\alpha}=2516 \mathrm{keV}\right)$ with $\mathrm{CdWO}_{4}$ detector [13] where it was observed at the first time $\left(T_{1 / 2}=1.1 \times 10^{18} \mathrm{yr}\right)$. Alpha particles from external ${ }^{241} \mathrm{Am}$ source (with a set of thin absorber 9 ) and from internal contamination of $\mathrm{CdWO}_{4}$ by U/Th chains were used; $E_{\alpha}=0.47-8.79 \mathrm{MeV}$. The experimental points and fitting curve calculated with Eq. (5) and $k B=10.1 \times 10^{-3} \mathrm{~g} \mathrm{MeV}^{-1} \mathrm{~cm}^{-2}$ are shown in Fig. $4 \mathrm{a}\left(\chi^{2} /\right.$ n.d.f. $=13$; without last point $\chi^{2} /$ n.d.f. $\left.=2.2\right)$.
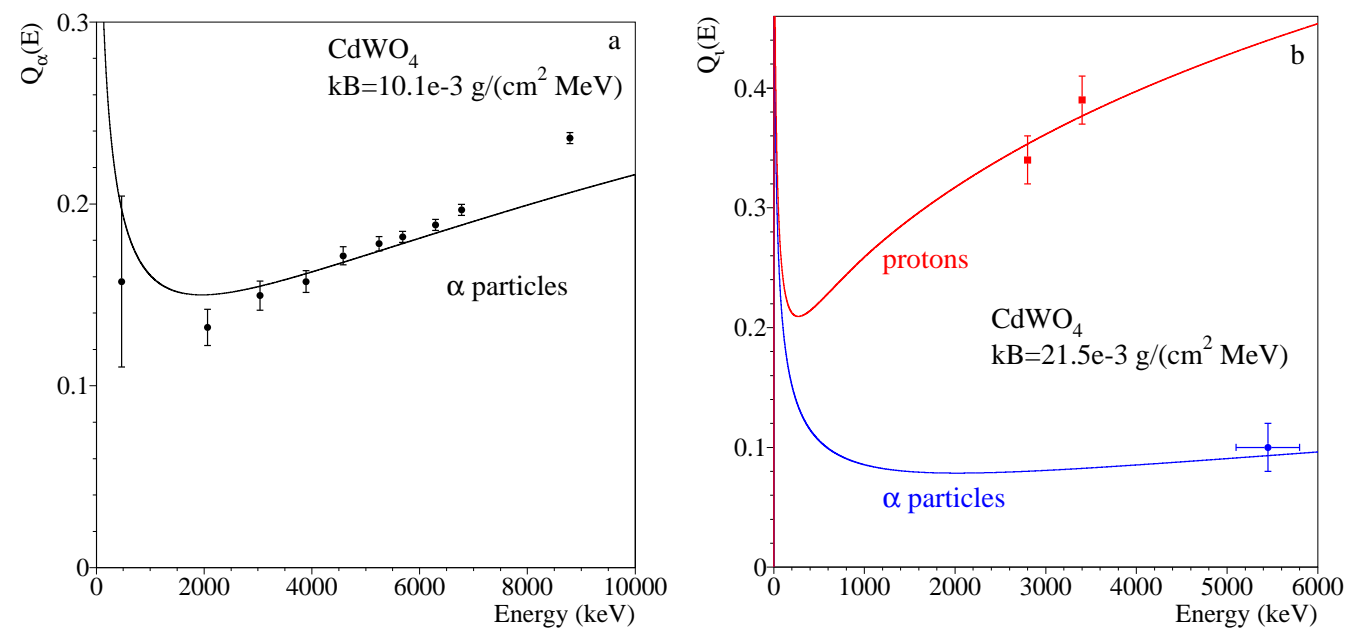

Fig. 4. (Color online) Quenching factors for $\alpha$ particles and protons in $\mathrm{CdWO}_{4}$. (a) Experimental data from Ref. [13] and their fit by Eq. (5) with $k B=10.1 \times 10^{-3}$ $\mathrm{g} \mathrm{MeV}^{-1} \mathrm{~cm}^{-2}$; (b) data for protons and $\alpha$ 's from [34] and their fit with $k B=21.5 \times 10^{-3} \mathrm{~g} \mathrm{MeV}^{-1} \mathrm{~cm}^{-2}$.

(2) In Ref. [34, quenching factors for protons provided by accelerator with $E_{p}=2.8 \mathrm{MeV}$ and $3.4 \mathrm{MeV}$ were measured as $0.34 \pm 0.02$ and $0.39 \pm 0.02$, respectively. Fit of these points by Eq. $(5)\left(\chi^{2} /\right.$ n.d.f. $\left.=0.90\right)$ was possible with the value of the Birks factor: $k B=21.5 \times 10^{-3} \mathrm{~g} \mathrm{MeV}^{-1} \mathrm{~cm}^{-2}$, very different from that obtained for $\mathrm{CdWO}_{4}$ by fitting data from Ref. [13. Quenching factors for $\alpha$ particles were also measured in [34. A mixed source with $\alpha$ 's from ${ }^{239} \mathrm{Pu}\left(E_{\alpha} \simeq 5.1 \mathrm{MeV}[35]\right),{ }^{241} \mathrm{Am}\left(E_{\alpha} \simeq 5.5 \mathrm{MeV}\right)$ and ${ }^{244} \mathrm{Cm}\left(E_{\alpha} \simeq 5.8\right.$

9 It should be noted that quenching factors in $\mathrm{CdWO}_{4}$ depend on direction of movement of $\alpha$ particle; we use here data for direction perpendicular to (010) crystal plane measured in wider energy range [13. 
$\mathrm{MeV}$ ) was used. Quenching factor of $Q_{\alpha} \simeq 0.1$ was measured (different from those in [13]). The value of $k B=21.5 \times 10^{-3} \mathrm{~g} \mathrm{MeV}^{-1} \mathrm{~cm}^{-2}$ obtained for protons gives theoretical curve for $\alpha$ 's in good agreement with this experimental result $\left(\chi^{2} /\right.$ n.d.f. $\left.=0.12\right)$, see Fig. 4 b.

\subsection{2 $\mathrm{CaF}(\mathrm{Eu})$}

(1) Quenching factors for $\alpha$ particles in $\mathrm{CaF}_{2}$ crystal scintillator $(\rho=3.18 \mathrm{~g}$ $\left.\mathrm{cm}^{-3}\right)$ doped by Eu at $0.4 \%$ were measured in [15] with external $\left({ }^{241} \mathrm{Am}\right)$ and internal (U/Th chains, $\left.{ }^{147} \mathrm{Sm}\right) \alpha$ sources $\left(E_{\alpha}=1-9 \mathrm{MeV}\right)$. The measured experimental points and their fit by Eq. (5) with the Birks factor $k B=5.3 \times$ $10^{-3} \mathrm{~g} \mathrm{MeV}^{-1} \mathrm{~cm}^{-2}$ are presented in Fig. 5a. Stopping powers of $\alpha$ particles were calculated with the SRIM and ASTAR codes; fitting with the ASTAR curve is better: $\chi^{2} /$ n.d.f. is 3.5 (with near $50 \%$ contribution from the last point) while for the SRIM $\chi^{2} /$ n.d.f. $=7.6$. It is a pity that the STAR package allows calculations of $d E / d r$ only for restricted list of materials in case of $\alpha$ particles and protons (and other ions are absent). From the other side, SRIM allows to calculate $d E / d r$ for any materials and for any ions, but not for electrons. Fig. 5 a gives an idea that probably calculations of $d E / d r$ for all particles inside the same package would give better description of quenching factors.
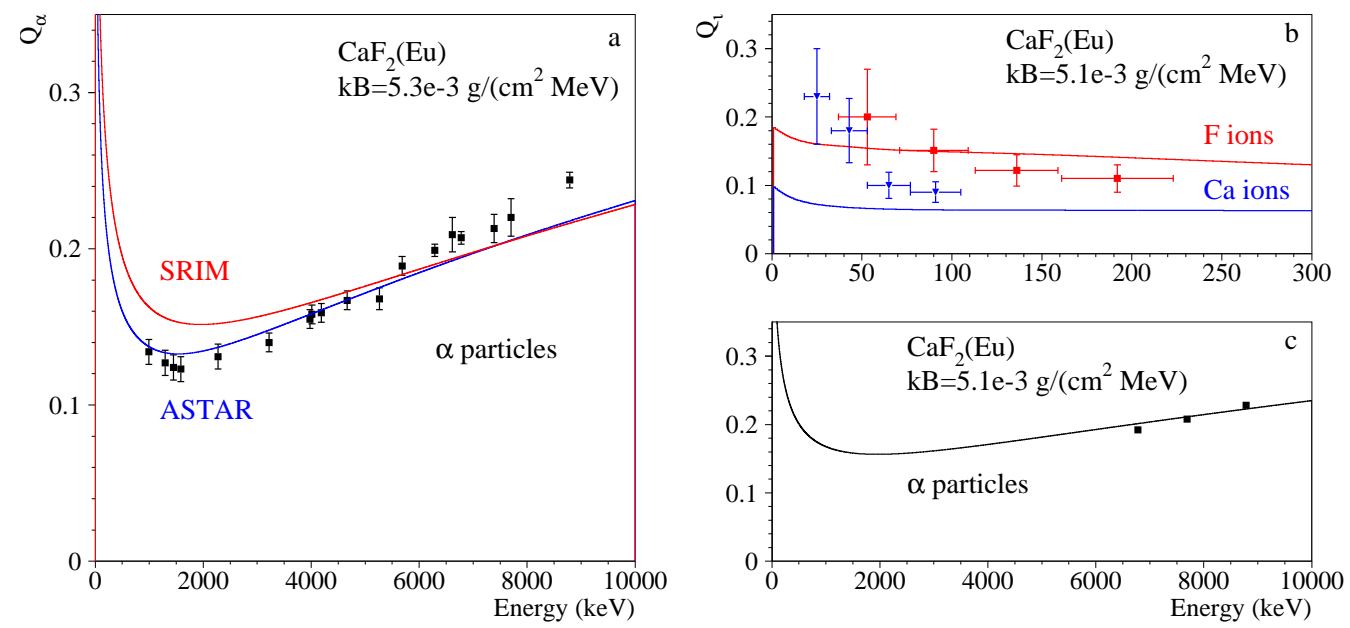

Fig. 5. (Color online) Quenching factors for $\alpha$ particles, and $\mathrm{F}$ and $\mathrm{Ca}$ ions in $\mathrm{CaF}_{2}(\mathrm{Eu})$. (a) Experimental data from Ref. 15] for $\alpha$ particles and their fit by Eq. (5) with $k B=5.3 \times 10^{-3} \mathrm{~g} \mathrm{MeV}^{-1} \mathrm{~cm}^{-2}$ with stopping powers calculated with the SRIM and ASTAR. (b) Data for F (squares) and Ca (triangles) ions from [36] and their fit with $k B=5.1 \times 10^{-3} \mathrm{~g} \mathrm{MeV}^{-1} \mathrm{~cm}^{-2}$. (c) Data from [37] for $\alpha$ 's with the same $k B$ value as in (b).

(2) Quenching factors for $\mathrm{F}$ and $\mathrm{Ca}$ ions in $\mathrm{CaF}_{2}(\mathrm{Eu})$ measured in [36] are shown in Fig. 5b. The $k B$ value was obtained by fitting the $\mathrm{F}$ data as: $k B=$ $5.1 \times 10^{-3} \mathrm{~g} \mathrm{MeV}^{-1} \mathrm{~cm}^{-2}\left(\chi^{2} /\right.$ n.d.f. $\left.=1.3\right)$. Curve for Ca ions was calculated with this $k B$; however, agreement with experimental points is not so good $\left(\chi^{2} /\right.$ n.d.f. $\left.=4.3\right)$. 
(3) Quenching factors for $\alpha$ particles in $\mathrm{CaF}_{2}(\mathrm{Eu})$, obtained by the same group as in Ref. [36], can be derived from their paper [37]. Alpha peak from ${ }^{216} \mathrm{Po}\left(E_{\alpha}=6.778 \mathrm{MeV}[35]\right)$ was observed at energy of $1.3 \mathrm{MeV}$, from ${ }^{214} \mathrm{Po}$ $\left(E_{\alpha}=7.687 \mathrm{MeV}\right)$ - at $1.6 \mathrm{MeV}$, and from ${ }^{212} \mathrm{Po}\left(E_{\alpha}=8.784 \mathrm{MeV}\right)$ - at $2.0 \mathrm{MeV}$; thus corresponding quenching factors are equal $0.192,0.208$ and 0.228 , respectively. Because we could expect the same (or similar) conditions of measurements and data treatment in both [36] and [37, the value of $k B=5.1 \times 10^{-3} \mathrm{~g} \mathrm{MeV}^{-1} \mathrm{~cm}^{-2}$ could fit the data for $\alpha$ 's as well. Such a curve is presented in Fig. 5c and is in good agreement with the experimental data.

\section{$3.2 .3 \mathrm{PbWO}_{4}$}

Quenching factors for $\alpha$ particles in $\mathrm{PbWO}_{4}$ crystal scintillator $(\rho=8.28 \mathrm{~g}$ $\mathrm{cm}^{-3}$ ) were studied in 38$]$ in the range of energies $E_{\alpha}=2.1-5.3 \mathrm{MeV}$. They are shown in Fig. 6 together with fit by Eq. (5) with $k B=10.5 \times 10^{-3} \mathrm{~g}$ $\mathrm{MeV}^{-1} \mathrm{~cm}^{-2}\left(\chi^{2} /\right.$ n.d.f. $\left.=3.3\right)$.

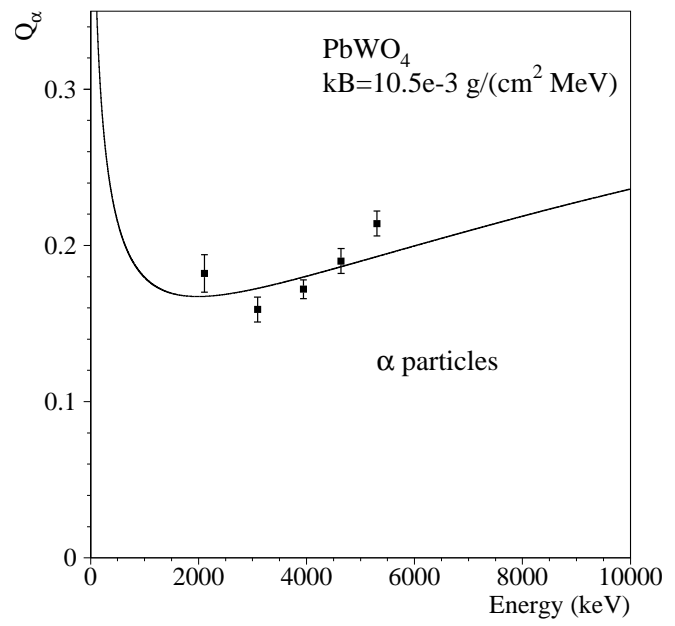

Fig. 6. Quenching factors for $\alpha$ particles in $\mathrm{PbWO}_{4}$ measured in [38] and their fit with $k B=10.5 \times 10^{-3} \mathrm{~g} \mathrm{MeV}^{-1} \mathrm{~cm}^{-2}$.

\subsection{4 $\mathrm{ZnWO}_{4}$}

(1) $\mathrm{ZnWO}_{4}\left(\rho=7.8 \mathrm{~g} \mathrm{~cm}^{-3}\right)$ is one of perspective scintillators in searches for dark matter particles (see e.g. [6]39]). Quenching factors for $\alpha$ particles were studied in $[40] 10$. They are shown in Fig. 7a together with fit by Eq. (5) with $k B=9.0 \times 10^{-3} \mathrm{~g} \mathrm{MeV}^{-1} \mathrm{~cm}^{-2}$; the agreement between the calculated curve and the experimental data is good $\left(\chi^{2} /\right.$ n.d.f. $\left.=0.93\right)$.

\footnotetext{
$\overline{{ }^{10} \mathrm{As} \text { in }} \mathrm{CdWO}_{4}$, quenching factors in $\mathrm{ZnWO}_{4}$ depend on direction of movement of $\alpha$ particle; we use here data for direction perpendicular to (010) crystal plane measured in wider energy range [40].
} 


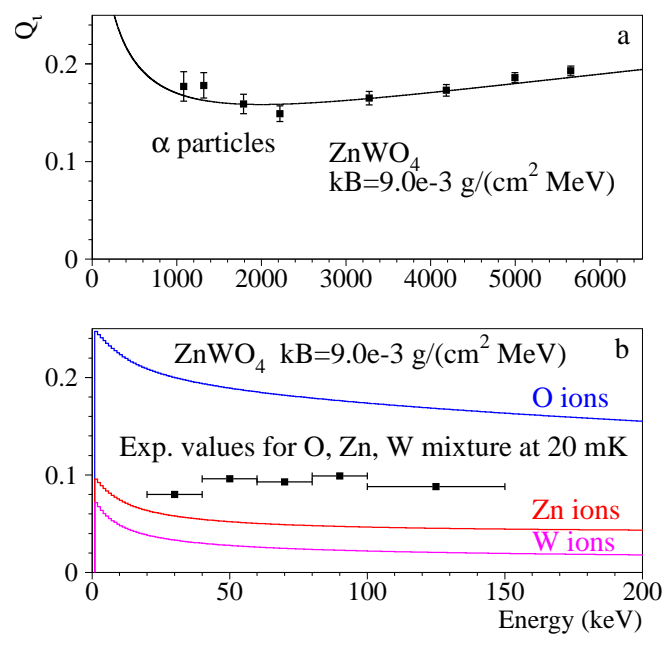

Fig. 7. (Color online) (a) Quenching factors for $\alpha$ particles in $\mathrm{ZnWO}_{4}$ measured in [40] and their fit with the Birks factor $k B=9.0 \times 10^{-3} \mathrm{~g} \mathrm{MeV}^{-1} \mathrm{~cm}^{-2}$. (b) Quenching factors for $\mathrm{O}, \mathrm{Zn}$ and $\mathrm{W}$ ions in $\mathrm{ZnWO}_{4}$ calculated with this $k B$ value together with experimental data for mixture of $\mathrm{O}, \mathrm{Zn}, \mathrm{W}$ factors measured at temperature $\simeq 20 \mathrm{mK}[6]$.

(2) In Fig. 7b, calculated quenching factors for $\mathrm{O}, \mathrm{Zn}$ and $\mathrm{W}$ ions obtained with this $k B$ value are presented. Also shown are experimental data collected with $\mathrm{ZnWO}_{4}$ detector in bolometric measurements [6]. Comparing calculations with these data, we have to remember that: (a) the latter are in fact data for some mixture of quenching factors for $\mathrm{O}, \mathrm{Zn}$ and $\mathrm{W}$ ions; (b) predictions are given for $k B=9.0 \times 10^{-3} \mathrm{~g} \mathrm{MeV}^{-1} \mathrm{~cm}^{-2}$ derived by fitting data for $\alpha$ particles measured at room temperature while in Ref. [6] temperature was $\simeq 20 \mathrm{mK}$. To obtain reliable predictions, data for all particles should be collected at the same conditions (and with the same data treatment).

\subsection{5 $\mathrm{CaWO}_{4}$}

Quenching factors for $\mathrm{CaWO}_{4}$ scintillator $\left(\rho=6.06 \mathrm{~g} \mathrm{~cm}^{-3}\right)$ probably are the most extensively investigated, in particular, because of numerous studies in the CRESST experimental searches for dark matter [41].

(1) Values for $\alpha$ particles were measured with external and internal $\alpha$ sources in [14] in the energy range $E_{\alpha} \simeq 0.5-8 \mathrm{MeV}$. These data are shown in Fig. 8 together with their fit by Eq. (5) with $k B=6.2 \times 10^{-3} \mathrm{~g} \mathrm{MeV}^{-1} \mathrm{~cm}^{-2}$ $\left(\chi^{2} /\right.$ n.d.f. $\left.=6.4\right)$.

(2) Energy dependence of quenching factors for O, Ca and $\mathrm{W}$ ions in $\mathrm{CaWO}_{4}$ was measured with monoenergetic neutron beam in [42] (for W, only upper limits of $Q_{i}$ were obtained, see Fig. 9a). Data for $\mathrm{O}$ ions were fitted by Eq. (5); the obtained value of the Birks factor is: $k B=8.0 \times 10^{-3} \mathrm{~g} \mathrm{MeV}^{-1} \mathrm{~cm}^{-2}$ (no surprise that it is different from that determined above for $\alpha$ particles due 


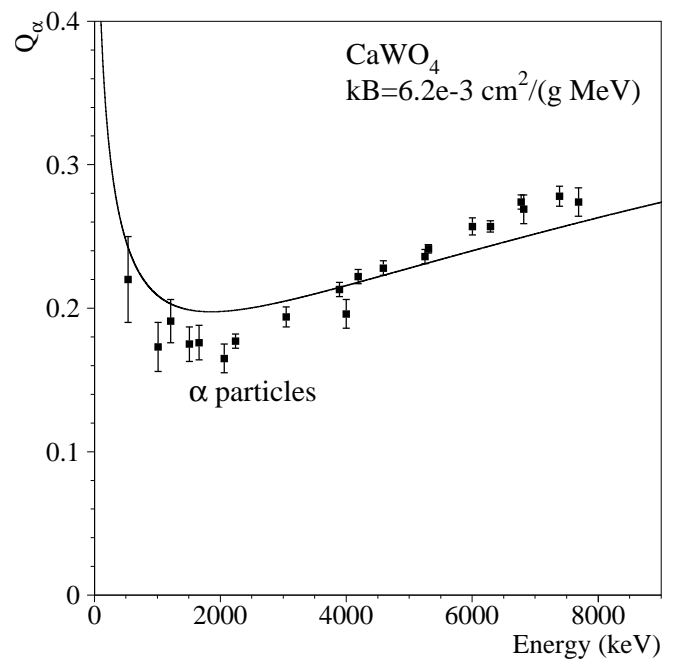

Fig. 8. Quenching factors for $\alpha$ particles in $\mathrm{CaWO}_{4}$ measured in [14] and their fit with $k B=6.2 \times 10^{-3} \mathrm{~g} \mathrm{MeV}^{-1} \mathrm{~cm}^{-2}$.

to different conditions of measurements and data treatment) 11 . Now, fixing this $k B$ value, we calculate quenching factors for $\mathrm{Ca}$ and $\mathrm{W}$ ions; all results are shown in Fig. 9a. One can say that calculations for $\mathrm{O}$ ions are in reliable agreement with the experimental points $\left(\chi^{2} /\right.$ n.d.f. $\left.=4.8\right)$, and also curve for $\mathrm{W}$ ions is not in contradiction with the measured $\mathrm{W}$ limits.
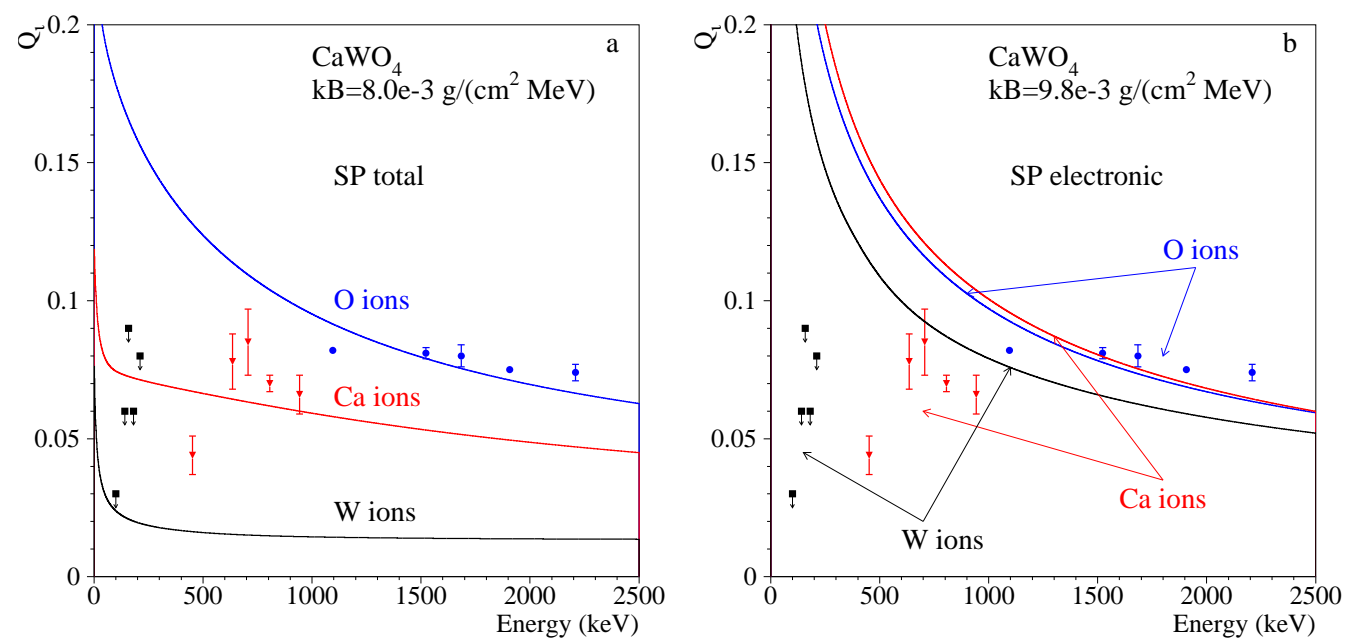

Fig. 9. (Color online) Quenching factors for $\mathrm{O}$ (circles), Ca (triangles) and $\mathrm{W}$ (squares) ions in $\mathrm{CaWO}_{4}$ measured in 42 and their fits: (a) with the ion total $\mathrm{SP}$ and $k B=8.0 \times 10^{-3} \mathrm{~g} \mathrm{MeV}^{-1} \mathrm{~cm}^{-2}$; (b) with only electronic part of the SP $\left(k B=9.8 \times 10^{-3} \mathrm{~g} \mathrm{MeV}^{-1} \mathrm{~cm}^{-2}\right)$.

(3) We want to return here to discussion on which stopping power (SP) - total

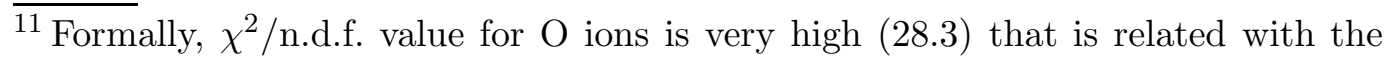
small error bars in the experimental data. However, it should be noted that deviations of the calculated values from the experimental ones are in the range of only 2 $-12 \%$. 
or only electronic part - is better to use in fitting experimental data. Using the same ideology, the experimental results for $\mathrm{O}$ ions were fitted by Eq. (5) where only electronic part of ion SP was taken into account. Obtained Birks factor was equal $k B=9.8 \times 10^{-3} \mathrm{~g} \mathrm{MeV}^{-1} \mathrm{~cm}^{-2}$; fit is worse than that with the total $\mathrm{SP}$ (see Fig. 9b). With this $k B$ value, quenching curves for $\mathrm{Ca}$ and $\mathrm{W}$ ions were calculated; they are in evident disagreement with the experimental data. Thus, use of the ion total SP in fitting and predicting quenching factors in the proposed approach allows to describe experimental data in a much better way.

(4) Dependence of the light output and quenching factors in $\mathrm{CaWO}_{4}$ on atomic mass of ion was measured in Ref. 43 by impinging various ions - from $\mathrm{H}$ to $\mathrm{Au}$ - onto the scintillating crystal. All ions had energy of $18 \mathrm{keV}$. Inverse values to the relative light outputs of Eq. (6), normalized to that of electrons at $6 \mathrm{keV}$, were calculated and presented in Table 4 of Ref. [43]:

$$
R_{i}^{\prime}=\frac{1}{R_{i}(E)}=\frac{L_{e}\left(E_{0}\right) / E_{0}}{L_{i}(E) / E}
$$

with $E=18 \mathrm{keV}$ and $E_{0}=6 \mathrm{keV}$; values of $R_{i}^{\prime}$ changed from $\simeq 2$ for $\mathrm{H}$ to $\simeq 40$ for $\mathrm{Au}$. Remembering that the $k B$ values could be different under different experimental conditions and data treatment, we will use $R_{i}^{\prime}$ for $\mathrm{H}$ to determine $k B$ in this particular measurements and will use this $k B$ value to calculate $R_{i}^{\prime}$ for all other ions. Value of $k B=17 \times 10^{-3} \mathrm{~g} \mathrm{MeV}^{-1} \mathrm{~cm}^{-2}$ gives $R_{\mathrm{H}}^{\prime}=2.17$ that well reproduces values for $\mathrm{H}$ given in Table 4 of 43 ] $(2.15 \pm 0.02$ for collection of the scintillation signal during $\Delta t=40 \mu \mathrm{s}$, and $2.18 \pm 0.02$ for $\Delta t=50 \mu \mathrm{s}) . R_{i}^{\prime}$ values for other ions calculated with this $k B$ value are shown in Fig. 10a together with experimental result 12 of Ref. [43] in dependence on ion's $Z$ number. Theoretical points lay on smooth curve well fitted by polynomial $R_{i}^{\prime}(Z)=a+b Z+c Z^{2}$ with $a=0.87098, b=0.98708$ and $c=-5.9896 \times 10^{-3}$ (also shown in Fig. 10a).

Comparison of the calculated and experimental values gives extremely high value of $\chi^{2} /$ n.d.f. $=112$ that is related mainly with deviations for O, Si, $\mathrm{Ca}, \mathrm{Cu}$ and especially $\mathrm{Y}$ points. It should be noted that the value for $\mathrm{Y}$ evidently deviates from the general trend; also error bars in [43] could be underestimated. F.e., new data for various ions are presented in Ref. [44] where points for $\mathrm{O}, \mathrm{Si}, \mathrm{Ca}, \mathrm{Cu}$ and $\mathrm{Y}$ are much lower (and in much better agreement with the calculated curve), while points for other ions are approximately as in Fig. 10a. However, because the data of Ref. [44] were preliminary and still are not explained in detail, we do not use them here.

\footnotetext{
${ }^{12}$ We use data for $\Delta t=50 \mu$ s that gives more complete collection of scintillating signal; however values for $\Delta t=40 \mu$ s are close.
} 

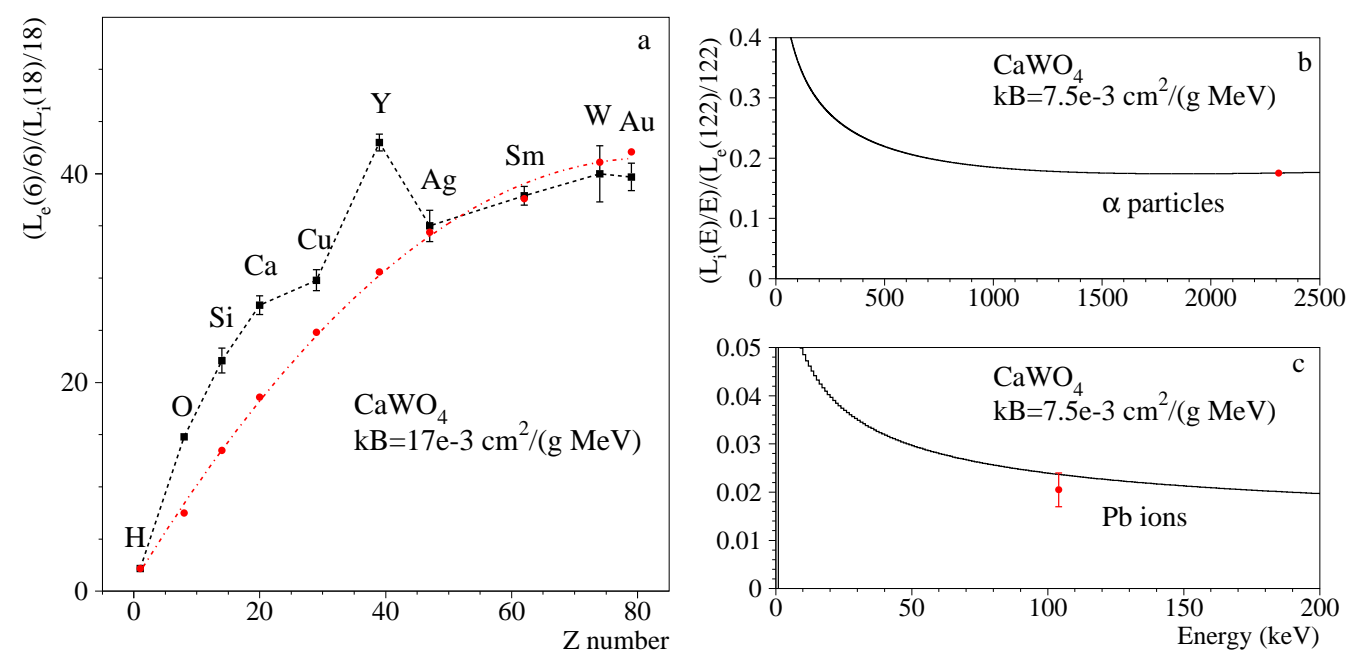

Fig. 10. (Color online) (a) Dependence of inverse of the relative light output $1 / R_{i}$ at $E_{i}=18 \mathrm{keV}$, normalized to that for electron at $E_{e}=6 \mathrm{keV}$ on ion's $Z$ number: squares are experimental points from [43, and circles are calculated values with $k B=17 \times 10^{-3} \mathrm{~g} \mathrm{MeV}^{-1} \mathrm{~cm}^{-2}$. (b) Relative light output for $\alpha$ particles with $k B=7.5 \times 10^{-3} \mathrm{~g} \mathrm{MeV}^{-1} \mathrm{~cm}^{-2}$ to fit experimental point at $2.3 \mathrm{MeV}$ measured at $7 \mathrm{mK}$ in [41. (c) Predicted curve for $\mathrm{Pb}$ ions with $k B$ the same as in (b) and experimental point from [41] also measured at $7 \mathrm{mK}$.

(5) Fig. 14 of Ref. [43] includes also $R_{i}^{\prime}$ values for $\alpha$ particles at $2.3 \mathrm{MeV}$ $\left({ }^{147} \mathrm{Sm}\right)$ and for $\mathrm{Pb}$ ions at $104 \mathrm{keV}$ (nuclear recoil after $\alpha$ decay of ${ }^{210} \mathrm{Po}$ ) measured in [41]. Taking into account that (a) quenching factors are energy dependent and (b) data for $\alpha$ and $\mathrm{Pb}$ were measured at a temperature $7 \mathrm{mK}$ [41], one could not expect perfect agreement between these $R_{i}^{\prime}$ and $R_{i}^{\prime}$ for all other ions taken in [43] at $18 \mathrm{keV}$ and measured at a room temperature. However, inside our ideology, data for $\alpha$ and $\mathrm{Pb}$, taken at the same conditions, should be self-consistent. Once more, we can use the relative light output for $\alpha$ particle $L_{\alpha}(E) / E$ at $2.3 \mathrm{MeV}$ normalized to that for electrons at $122 \mathrm{keV}$ (as in Ref. [41 13 ) to determine the Birks factor; it gives $k B=7.5 \times 10^{-3} \mathrm{~g}$ $\mathrm{MeV}^{-1} \mathrm{~cm}^{-2}$ (see Fig. 10b). Curve with this $k B$ value for $\mathrm{Pb}$ ions is shown in Fig. 10c; at energy of $104 \mathrm{keV}$ it agrees with quenching factor given in [41,43] $\left(\chi^{2} /\right.$ n.d.f. $\left.=0.78\right)$. Taking into account big difference in atomic numbers of $\alpha$ particle and $\mathrm{Pb}$ ion (2 and 82 , respectively), as well as in their energies (2.3 $\mathrm{MeV}$ and $104 \mathrm{keV}$ ), this example demonstrates consistency in description of such diverse data.

\subsection{6 $\mathrm{CsI}(\mathrm{Tl})$ and $\mathrm{CsI}(\mathrm{Na})$}

(1) Experimental data for quenching factors of Cs and I ions in CsI(Tl) crystal scintillators $\left(\rho=4.51 \mathrm{~g} \mathrm{~cm}^{-3}\right)$ published in [45], as well as in [46] (KIMS

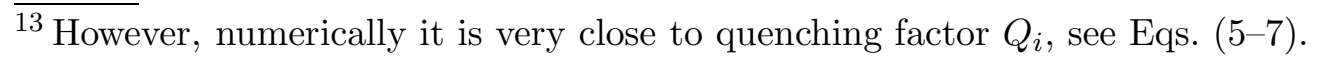


collaboration) 14 and [47] (TEXONO collaboration) are shown in Fig. 11a, $11 \mathrm{~b}$ and 11c, respectively; total energy range of ions was 7 to $135 \mathrm{keV}$. All these data sets are well described by Eq. (5) with the same value of the Birks factor: $k B=3.2 \times 10^{-3} \mathrm{~g} \mathrm{MeV}^{-1} \mathrm{~cm}^{-2}\left(\chi^{2} /\right.$ n.d.f. value is $1.9,0.51$ and 0.49 , respectively). Cs and I nuclei have very close atomic numbers and masses, and their quenching factors also are very close (see Fig. 11a).
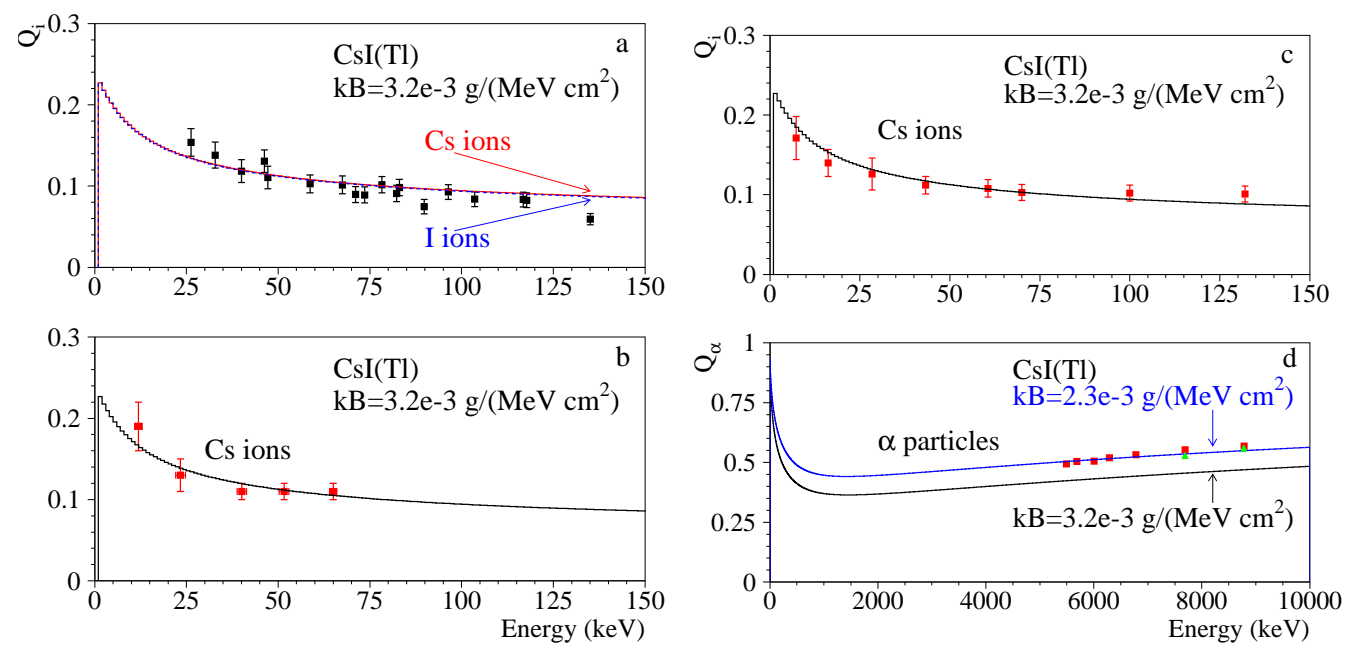

Fig. 11. (Color online) Quenching factors (squares) for Cs and I ions in $\mathrm{CsI}(\mathrm{Tl})$ measured in: [45] (a), 46] (b), 47] (c) and their fit with the Birks factor $k B=3.2 \times 10^{-3}$ $\mathrm{g} \mathrm{MeV}^{-1} \mathrm{~cm}^{-2}$; (d) quenching factors for $\alpha$ particles in $\mathrm{CsI}(\mathrm{Tl})$ measured in 48, (triangles) and [49] (squares) and calculated quenching curves with $k B=3.2 \times 10^{-3}$ g $\mathrm{MeV}^{-1} \mathrm{~cm}^{-2}$ and $k B=2.3 \times 10^{-3} \mathrm{~g} \mathrm{MeV}^{-1} \mathrm{~cm}^{-2}$.

(2) The KIMS and TEXONO collaborations measured also quenching factors for $\alpha$ particles derived from studies of internal contamination of $\mathrm{CsI}(\mathrm{Tl})$ detectors [48,49]. Their values are consistent; see Fig. 11d where they are presented together with a calculated quenching curve for $\alpha$ particles with the same $k B=3.2 \times 10^{-3} \mathrm{~g} \mathrm{MeV}^{-1} \mathrm{~cm}^{-2}$ as above for Cs and I ions. However, this curve is lower than the experimental points which are much better described by curve with $k B=2.3 \times 10^{-3} \mathrm{~g} \mathrm{MeV}^{-1} \mathrm{~cm}^{-2}$. This could be some underestimation of $Q_{\alpha}$ values in our approach but as well we have to remember that the data for Cs and I were taken in devoted measurements with neutron beams while data for $\alpha$ particles were collected in separate measurements with probably non-identical experimental conditions and details of data treatment (temperature in the $\mathrm{Cs} / \mathrm{I}$ measurements by $\mathrm{KIMS}$ was $24.5^{\circ} \mathrm{C}$ in [46], and $26-29^{\circ} \mathrm{C}$ in $\alpha$ measurements [48, etc.).

(3) Experimental quenching factors for Cs and I ions in CsI(Na) crystal scintillators measured in [46] are shown in Fig. 12a (data for $0.0188 \%$ of Na were taken but results for other $\mathrm{Na}$ amounts are similar). Data points are fitted

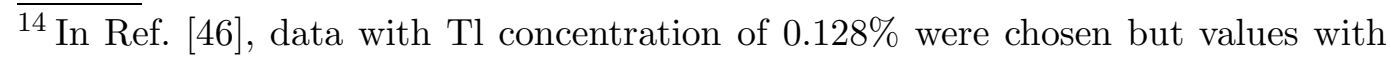
other $\mathrm{Tl}$ concentrations are similar. 
by Eq. (5) calculated with $k B=5.5 \times 10^{-3} \mathrm{~g} \mathrm{MeV}^{-1} \mathrm{~cm}^{-2}\left(\chi^{2} /\right.$ n.d.f. $\left.=1.9\right)$. Quenching factors for $\alpha$ particles obtained with this $k B$ value are shown in Fig. 12b.

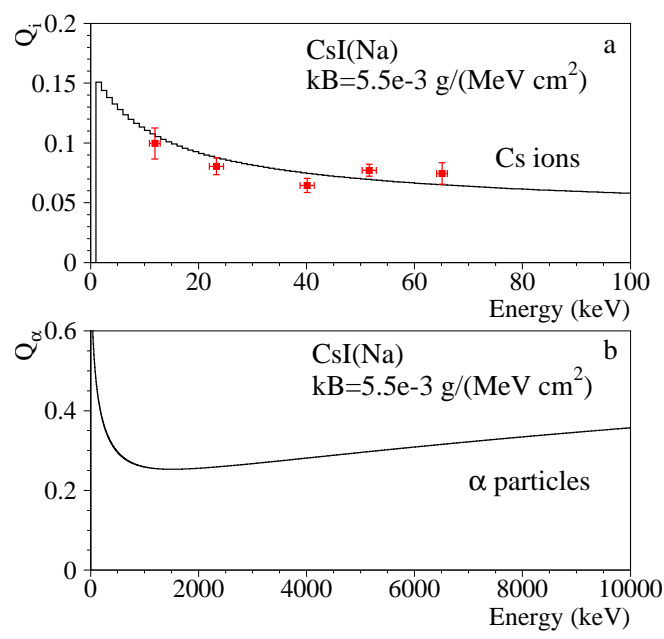

Fig. 12. (Color online) (a) Quenching factors for Cs and I ions in $\operatorname{CsI}(\mathrm{Na})$ measured in [46] and their fit by Eq. (5) with $k B=5.5 \times 10^{-3} \mathrm{~g} \mathrm{MeV}^{-1} \mathrm{~cm}^{-2}$. (b) Quenching factors for $\alpha$ particles in $\operatorname{CsI}(\mathrm{Na})$ calculated with the $k B$ value as in (a).

\subsection{7 $\mathrm{NaI}(\mathrm{Tl})$}

(1) Quenching factors for $\mathrm{Na}$ and I nuclei in $\mathrm{NaI}(\mathrm{Tl})$ crystal scintillators $\left(\rho=3.67 \mathrm{~g} \mathrm{~cm}^{-3}\right)$ measured in Ref. [50] in energy range of $7-215 \mathrm{keV}$ and $13-54 \mathrm{keV}$, respectively, are presented in Fig. 13a. Value of the Birks factor which allows to describe curve for $\mathrm{Na}$ ions with Eq. (5) is equal: $k B=3.8 \times 10^{-3}$ $\mathrm{g} \mathrm{MeV}^{-1} \mathrm{~cm}^{-2}\left(\chi^{2} /\right.$ n.d.f. $\left.=1.2\right)$. Once found, it also allows to calculate quenching curve for I ions, and this curve is in excellent agreement $\left(\chi^{2} /\right.$ n.d.f. $\left.=0.26\right)$ with the experimental data, as one can see in Fig. 13a.

(2) In recent work [8], quenching factors for $\mathrm{Na}$ ions in $\mathrm{NaI}(\mathrm{Tl})$ were measured in more detail in low energy region $(10-100 \mathrm{keV})$. Obtained experimental values are lower than those in [50]. This demands higher $k B$ factor to describe higher quenching: $k B=6.5 \times 10^{-3} \mathrm{~g} \mathrm{MeV}^{-1} \mathrm{~cm}^{-2}$. Corresponding calculated curve well describes experimental values (except of the last point at $100 \mathrm{keV}$ ), see Fig. $13 \mathrm{~b}\left(\chi^{2} /\right.$ n.d.f. is equal 4.0 for all the points and 0.56 with the last point excluded). Calculations for I ions are also presented (however quenching factors for I were not measured in [8]).

It is worth noting that with the $k B$ values as in Figs. 13a and 13b, our suggested procedure predicts a quenching factor for $6 \mathrm{MeV} \alpha$ particles of 0.35 and 0.25 , respectively; these values are lower by a factor $1.5-2$ than those typically measured (however, under different experimental conditions) for $\mathrm{NaI}(\mathrm{Tl})$ (see f.e. [51]). 

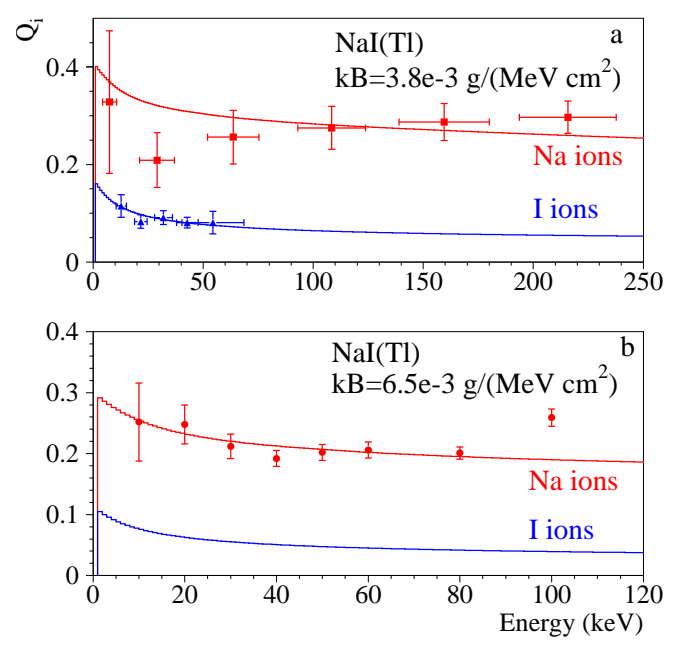

Fig. 13. (Color online) (a) Quenching factors for Na (squares) and I (triangles) in $\mathrm{NaI}(\mathrm{Tl})$ measured in [50] and calculated curves with $k B=3.8 \times 10^{-3} \mathrm{~g} \mathrm{MeV}^{-1}$ $\mathrm{cm}^{-2}$. (b) $Q_{i}$ values for Na ions from [8] and fitting curve with $k B=6.5 \times 10^{-3} \mathrm{~g}$ $\mathrm{MeV}^{-1} \mathrm{~cm}^{-2}$. Prediction for I ions is also shown.

(3) Quenching factors for $\mathrm{Na}$ and I ions in $\mathrm{NaI}(\mathrm{Tl})$ scintillators were also measured in other works with monoenergetic neutron beams as:

$-Q_{\mathrm{Na}}(E \simeq 18-800 \mathrm{keV}) \simeq 0.30$, and $Q_{\mathrm{I}}(E \simeq 20-120 \mathrm{keV}) \simeq 0.1$ (with some increase at lower energies) [52];

$-Q_{\mathrm{Na}}(E \simeq 18-74 \mathrm{keV})=0.25 \pm 0.03$, and $Q_{\mathrm{I}}(E \simeq 40-100 \mathrm{keV})=0.08 \pm 0.02$ [53];

$-Q_{\mathrm{Na}}(E \simeq 50-336 \mathrm{keV})$, values are consistent with constant of $0.27 \pm 0.02$ [54].

In addition, in measurements with ${ }^{252} \mathrm{Cf}$ source quenching factors in some effective energy range were obtained as:

$-Q_{\mathrm{Na}}(E=5-100 \mathrm{keV})=0.40 \pm 0.20$, and $Q_{\mathrm{I}}(E \simeq 40-300 \mathrm{keV})=0.05 \pm 0.02$ [55];

$-Q_{\mathrm{Na}}(E=7-100 \mathrm{keV})=0.30$, and $Q_{\mathrm{I}}(E \simeq 20-330 \mathrm{keV})=0.09$ [56].

Comparing energy ranges investigated in these works with behaviour of quenching curves in Figs. 13a,b, one can note that the energy thresholds were not low enough to observe increase of quenching factors at lower energies which is predicted in our approach, and at higher energies $Q_{i}$ are consistent with constant values (taking into account experimental uncertainties). However, works [8,50] with lower thresholds give experimental values consistent with this prediction.

(4) In Ref. [57], energy dependence of quenching factor for $\alpha$ particles in range of energies $E_{\alpha}=5.7-6.8 \mathrm{MeV}$ (from internal contamination of one of $\mathrm{NaI}(\mathrm{Tl}$ ) crystals) was obtained as: $Q_{\alpha}\left(E_{\alpha}\right)=0.467(6)+0.0257(10) \times E_{\alpha}$, where $E_{\alpha}$ is in $\mathrm{MeV}$. This energy dependence (shown in Fig. 14a) can be reproduced by Eq. (5) with the Birks factor $k B=1.25 \times 10^{-3} \mathrm{~g} \mathrm{MeV}^{-1} \mathrm{~cm}^{-2}$, much lower than those found for data of Refs. [8 50] in Figs. 13a,b. 


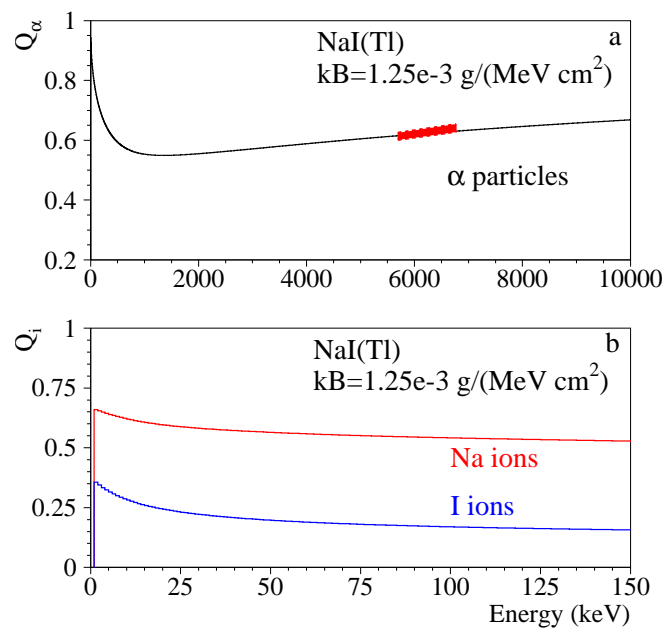

Fig. 14. (Color online) (a) Quenching factor for $\alpha$ particles measured in [57] (filled area represents corridor of uncertainties) and its fit with $k B=1.25 \times 10^{-3} \mathrm{~g} \mathrm{MeV}^{-1}$ $\mathrm{cm}^{-2}$. (b) Predicted quenching curves for $\mathrm{Na}$ and I ions with $k B=1.25 \times 10^{-3} \mathrm{~g}$ $\mathrm{MeV}^{-1} \mathrm{~cm}^{-2}$. Note that the calculated $Q_{\mathrm{Na}}$ and $Q_{\mathrm{I}}$ are $\simeq 2$ higher than those measured in the DAMA [56] and other experiments - however, under different conditions.

Following our method, we predict quenching factors for $\mathrm{Na}$ and I ions in this crystal with the obtained $k B$ value; they are shown in Fig. $14 \mathrm{~b}$ and are significantly higher (around 0.6 for $\mathrm{Na}$, and 0.3 for I) than the ones measured by the DAMA group in [56] (0.30 and 0.09, respectively), which are also similar to other determinations available in literature.

Considering that our suggested procedure predicts: i) for the cases of Refs. [8,50] a quenching factor for $\alpha$ particles in the $\mathrm{MeV}$ region lower by a factor $1.5-2$ than those typically measured; ii) quenching factors for $\mathrm{Na}$ and I ions at low energy higher by a factor about 2 than those measured by [56] and other experiments; one could consider the obtained here results with some cautious attitude. However, we want to once more remind that quenching factors for $\mathrm{Na} / \mathrm{I}$ ions and $\alpha$ particles were measured in both cases under different experimental conditions. It would be of great interest to measure them simultaneously, with the same data taking and treatment. Fig. 10 for $\mathrm{CaWO}_{4}$ and Fig. 13a for $\mathrm{NaI}(\mathrm{Tl})$ give examples that, if such a condition is fulfilled, the method gives self-consistent description of data for ions with very different $(A, Z)$ values.

As general consideration, it is worth noting that higher quenching factors would always be of big importance for searches of dark matter particles because, with a fixed energy threshold of a detector, higher quenching factors allow to study lower energies of DM particles. For example, when assuming some cases for WIMPs, the energy distribution would drop quasi-exponentially with energy and, thus, this would lead to higher experimental sensitivities in the DM searches. 


\subsection{8 $\mathrm{CeF}_{3}$}

Quenching factors for $\alpha$ particles in $\mathrm{CeF}_{3}$ crystal scintillator $\left(\rho=6.16 \mathrm{~g} \mathrm{~cm}^{-3}\right)$ were studied in [58] in the range of energies $E_{\alpha}=2.1-8.8 \mathrm{MeV}$. They are shown in Fig. 15 together with fit by Eq. (5) with $k B=11.1 \times 10^{-3} \mathrm{~g} \mathrm{MeV}^{-1}$ $\mathrm{cm}^{-2}\left(\chi^{2} /\right.$ n.d.f. $\left.=1.8\right)$.

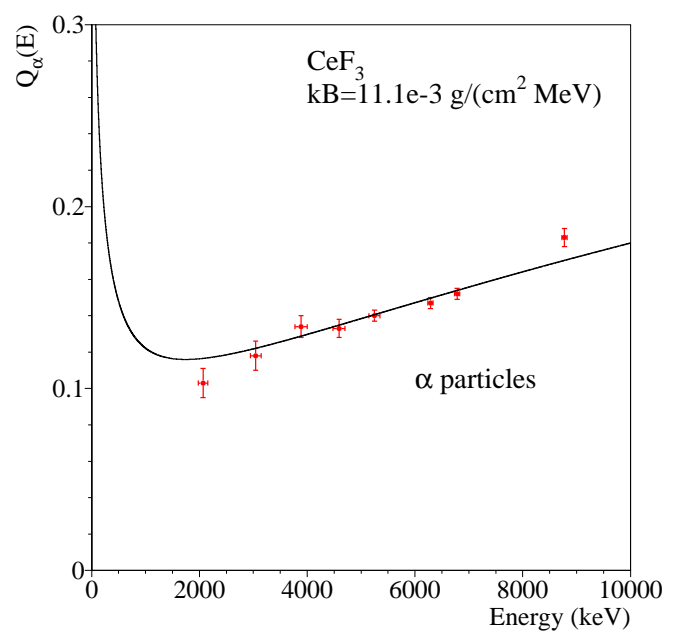

Fig. 15. (Color online) Quenching factors for $\alpha$ particles in $\mathrm{CeF}_{3}$ measured in 58 and their fit with $k B=11.1 \times 10^{-3} \mathrm{~g} \mathrm{MeV}^{-1} \mathrm{~cm}^{-2}$.

\subsection{Liquid noble gases}

\subsubsection{Liquid $X e$}

(1) Some data on quenching factors in liquid Xe scintillator (LXe, $\rho=3.52$ $\mathrm{g} \mathrm{cm}^{-3}$ at $-109^{\circ} \mathrm{C}[59 \sqrt{15}$ ) also support predicted in the described approach increase of $Q_{i}$ values at low energies. Results obtained in Ref. [60] are shown in Fig. 16a; they are well described by Eq. (5) with the Birks factor $k B=$ $3.5 \times 10^{-4} \mathrm{~g} \mathrm{MeV}^{-1} \mathrm{~cm}^{-2}\left(\chi^{2} /\right.$ n.d.f. $\left.=0.36\right)$. On the contrary, the Lindhard's theory [18] predicts decrease of $Q_{i}$ values at low energies; it is also shown in Fig. 16a (calculated using description in [20]).

(2) Quenching factors for Xe ions in LXe measured in [61] 16 are shown in Fig. 16b. They are much lower than those of Ref. [60], and to reproduce these

\footnotetext{
${ }^{15}$ Density of liquid Xe depends on pressure and temperature. However, numerical value of the Birks factor $k B$ does not depend on value of density if $k B$ is measured in $\mathrm{g} \mathrm{MeV}^{-1} \mathrm{~cm}^{-2}$.

${ }^{16}$ More exactly, relative light outputs $L_{i}(E) / E$ normalized to that for electrons of $122 \mathrm{keV}$ were measured in 61]; however they are very close here to quenching factors, see Eqs. (5-7).
} 

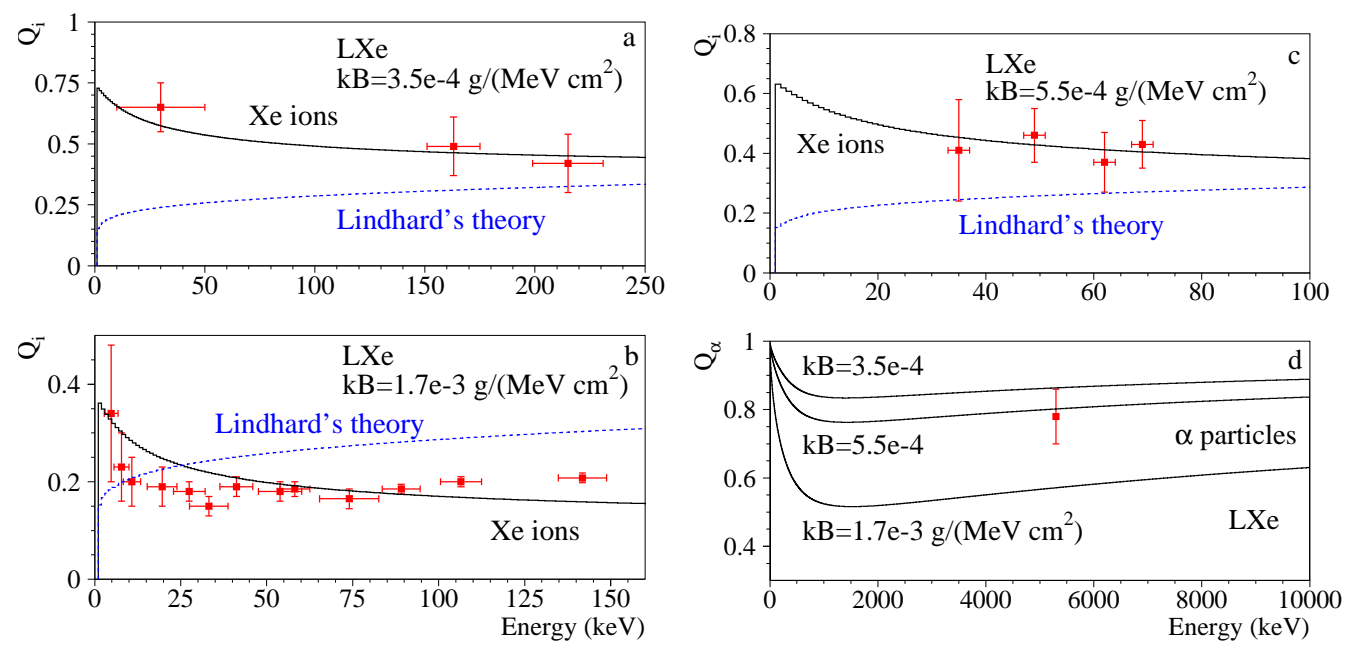

Fig. 16. (Color online) Quenching factors for Xe ions in LXe: (a) measured in 60] and fitting curve with $k B=3.5 \times 10^{-4} \mathrm{~g} \mathrm{MeV}^{-1} \mathrm{~cm}^{-2}$; (b) data from [61] and fitting curve with $k B=1.7 \times 10^{-3} \mathrm{~g} \mathrm{MeV}^{-1} \mathrm{~cm}^{-2}$; (c) measured in 62 and calculated curve with $k B=5.5 \times 10^{-4} \mathrm{~g} \mathrm{MeV}^{-1} \mathrm{~cm}^{-2}$. Lindhard's theoretical prediction is shown by dashed line in (a)-(c). (d) $Q_{\alpha}$ quenching curves calculated with different $k B$ values together with experimental point for $5.3 \mathrm{MeV} \alpha$ particle from 63 .

data with high quenching, the Birks factor also should have bigger value: $k B=1.7 \times 10^{-3} \mathrm{~g} \mathrm{MeV}^{-1} \mathrm{~cm}^{-2}\left(\chi^{2} /\right.$ n.d.f. $\left.=5.0\right)$.

(3) Experimental data from [62], measured in not so wide energy range (35-69 $\mathrm{keV}$ ), are consistent with a constant value (Fig. 16c). However, as well they are in good agreement with description by Eq. (5) with the Birks factor $k B=$ $5.5 \times 10^{-4} \mathrm{~g} \mathrm{MeV}^{-1} \mathrm{~cm}^{-2}\left(\chi^{2} /\right.$ n.d.f. $\left.=0.15\right)$.

(4) Quenching curves for $\alpha$ particles calculated with the three above quoted $k B$ values are shown in Fig. 16d together with experimental result of Ref. 63. for $5.3 \mathrm{MeV} \alpha$ particles: $Q_{\alpha}=0.78 \pm 0.08$. Because data of Ref. [63] were obtained in experimental conditions different from those in [60,61,62], no surprise that not all curves are in good agreement with the experimental point: to be in agreement, quenching factors for all particles should be obtained with the same detector and in the same experimental conditions and data treatment.

(5) It should be noted also that there are other experimental data sets for quenching factors of Xe ions in LXe which, on contrary, demonstrate decrease of $Q_{i}$ values at lower energies [64]. Results of Refs. [65, 66] were measured in energy range of $\simeq 47-110 \mathrm{keV}$ and $\simeq 43-65 \mathrm{keV}$, respectively, and are not far from constant values, as also could be expected from Figs. 16a-c. 


\subsubsection{Liquid Ar}

Data for liquid argon $\left(\rho=1.40 \mathrm{~g} \mathrm{~cm}^{-3}\right)$ are rather scarce. In fact, only one measurement was performed in the framework of the WARP project [67] where quenching factor for Ar ions was obtained with monoenergetic neutrons of 14 $\mathrm{MeV}$, however without fixing the angle of neutron scattering. Thus quenching factor was obtained for some effective energy range, with mean value of $65 \mathrm{keV}$, as: $Q_{\mathrm{Ar}}(E \simeq 65 \mathrm{keV})=0.28 \pm 0.03$ (at applied electric field of $1 \mathrm{kV} / \mathrm{cm}$ ). This point can be reproduced by Eq. (5) with the Birks factor of $k B=1.25 \times 10^{-3}$ $\mathrm{g} \mathrm{MeV}^{-1} \mathrm{~cm}^{-2}$; see Fig. 17a, where also curve calculated in the Lindhard's theory [18,20] is shown.
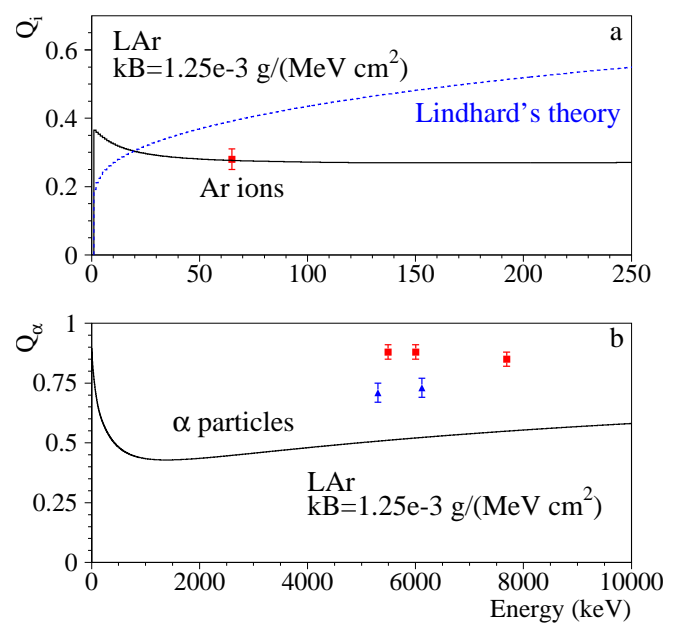

Fig. 17. (Color online) (a) Quenching factor for Ar ions in LAr measured in 67. and fitting curve with $k B=1.25 \times 10^{-3} \mathrm{~g} \mathrm{MeV}^{-1} \mathrm{~cm}^{-2}$. Lindhard's theoretical prediction is shown by dashed line. (b) $Q_{\alpha}$ quenching curve for LAr calculated with $k B=1.25 \times 10^{-3} \mathrm{~g} \mathrm{MeV}^{-1} \mathrm{~cm}^{-2}$ and experimental data measured in 68] (triangles) and 69] (squares).

Dependence of quenching factor for $\alpha$ particles on energy, calculated with this $k B$ value, is shown in Fig. 17b. Experimental values of $Q_{\alpha}$ measured in experiments [68,69] also are given; however, because they were obtained in different experimental conditions, they are not obliged to lay on the calculated curve with $k B=1.25 \times 10^{-3} \mathrm{~g} \mathrm{MeV}^{-1} \mathrm{~cm}^{-2}$, and even are not obliged to be in agreement between themselves (as one can see in Fig. 17b).

\subsubsection{Liquid Ne}

Experimental situation for liquid neon $\left(\rho=1.21 \mathrm{~g} \mathrm{~cm}^{-3}\right)$ is even worse than for LAr: to-date is only one experiment [11] where the relative light output normalized to that for electrons of $E_{0}=511 \mathrm{keV}$ was measured at the energy $E=387 \pm 11 \mathrm{keV}$ as: $\left(L_{\mathrm{Ne}}(E) / E\right) /\left(L_{e}\left(E_{0}\right) / E_{0}\right)=0.26 \pm 0.03$. Calculated quenching curve of Eq. (6) which is normalized to this experimental point is 
shown in Fig. $18 \mathrm{a}\left(k B=2.0 \times 10^{-3} \mathrm{~g} \mathrm{MeV}^{-1} \mathrm{~cm}^{-2}\right)$; Fig. 18b shows prediction for quenching for $\alpha$ particles calculated with this $k B$ factor.
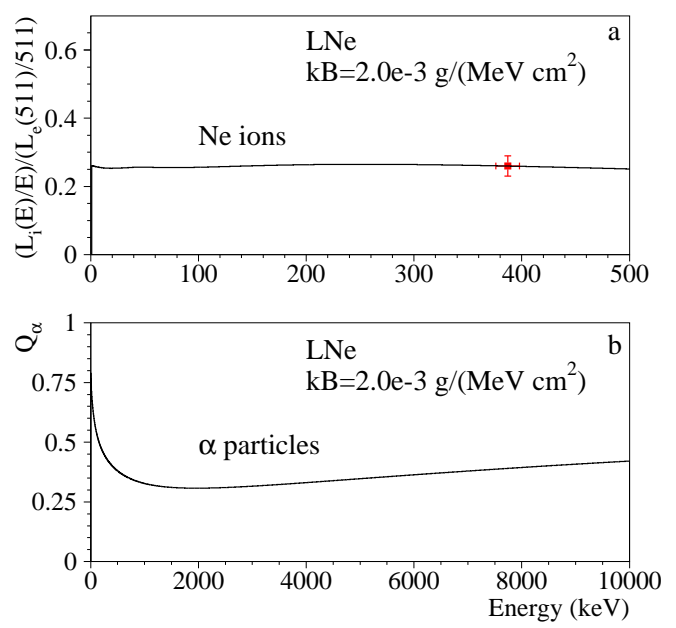

Fig. 18. (Color online) (a) Relative light output for Ne ions in liquid Ne. Curve is normalized to experimental value at $387 \mathrm{keV}$ from Ref. [1] $\left(k B=2.0 \times 10^{-3} \mathrm{~g}\right.$ $\mathrm{MeV}^{-1} \mathrm{~cm}^{-2}$ ). (b) Predicted quenching curve for $\alpha$ 's in LNe with $k B=2.0 \times 10^{-3}$ $\mathrm{g} \mathrm{MeV}^{-1} \mathrm{~cm}^{-2}$.

\subsection{Estimation of quenching factors for nuclear recoils in some scintillators}

While quenching factors for nuclear recoils that constitute such scintillators as $\mathrm{C}_{9} \mathrm{H}_{12}, \mathrm{CaF}_{2}(\mathrm{Eu}), \mathrm{ZnWO}_{4}, \mathrm{CaWO}_{4}, \mathrm{CsI}(\mathrm{Tl}), \mathrm{NaI}(\mathrm{Tl})$, LXe, LAr, LNe can be found above, for some other scintillators, which are considered as perspective detectors in the DM searches, quenching factors were not measured to-date. Below we give estimation of $Q_{i}$ values for nuclear recoils in $\mathrm{CdWO}_{4}, \mathrm{PbWO}_{4}$, $\mathrm{CeF}_{3}, \mathrm{Bi}_{4} \mathrm{Ge}_{3} \mathrm{O}_{12}, \mathrm{LiF}$ and ZnSe scintillators which are based on measured quenching factors for $\alpha$ particles in these materials. We should remember, of course, that the $k B$ values and thus quenching factors for $\alpha$ particles and recoils can be different for the same material in different conditions of measurements and data treatment. Nevertheless, results given below could be useful as providing some initial values of $Q_{i}$.

(1) Quenching factors for $\mathrm{O}, \mathrm{Cd}$ and $\mathrm{W}$ ions in $\mathrm{CdWO}_{4}$ scintillator are shown in Fig. 19a for two extreme $k B$ values: $k B=10.1 \times 10^{-3} \mathrm{~g} \mathrm{MeV}^{-1} \mathrm{~cm}^{-2}$ and $k B=21.5 \times 10^{-3} \mathrm{~g} \mathrm{MeV}^{-1} \mathrm{~cm}^{-2}$. The first $k B$ value was obtained by fitting experimental data of [13] for $\alpha$ particles (see Fig. 4a), and the second one by fitting data of [34] for $\alpha$ particles and protons (Fig. 4b).

(2) To calculate $Q_{i}$ for $\mathrm{O}, \mathrm{W}$ and $\mathrm{Pb}$ ions in $\mathrm{PbWO}_{4}$ (Fig. 19b), we use the value of $k B=10.5 \times 10^{-3} \mathrm{~g} \mathrm{MeV}^{-1} \mathrm{~cm}^{-2}$ obtained by fitting data of [38] for $\alpha$ particles (see Fig. 6). 

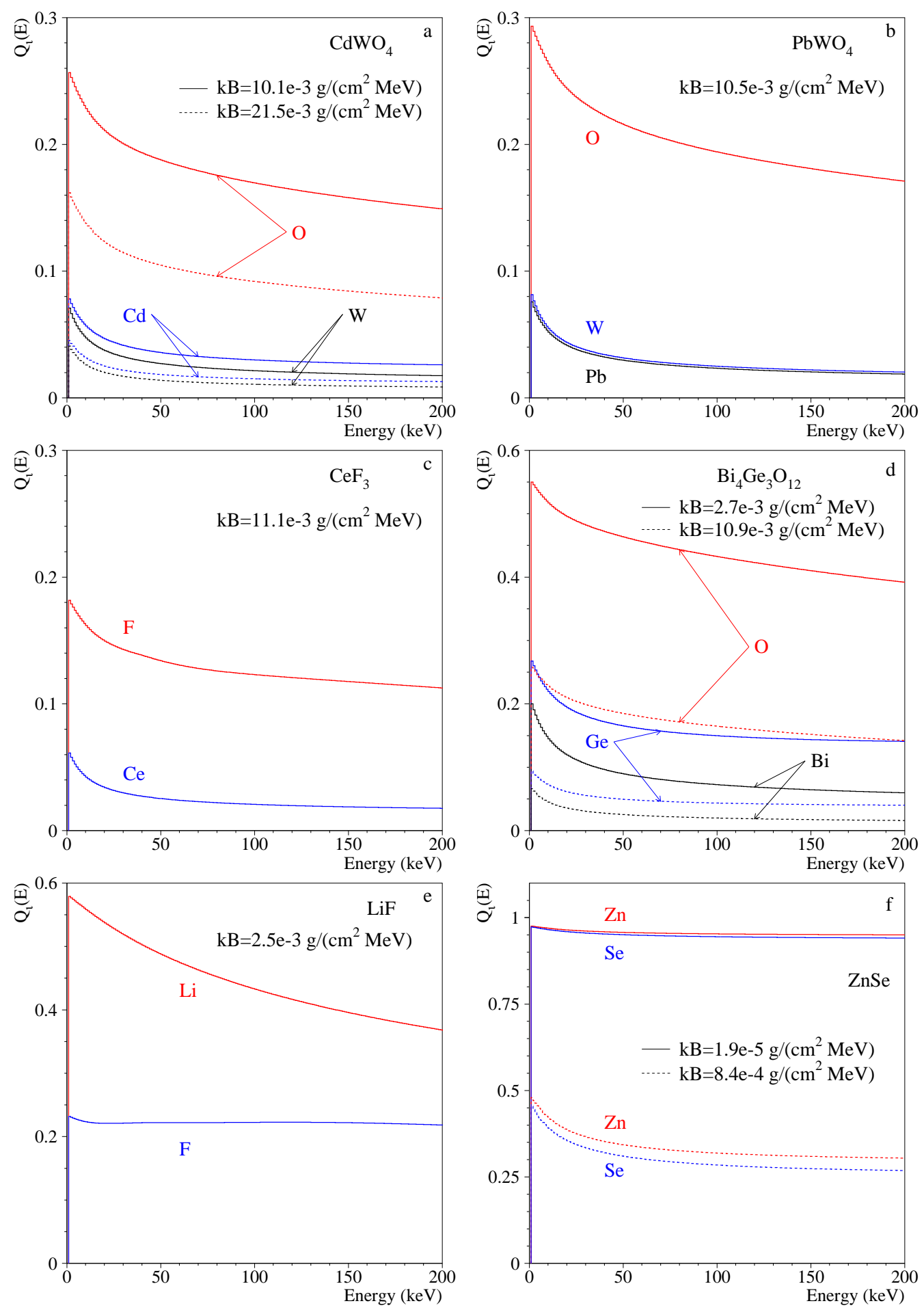

Fig. 19. (Color online) Quenching factors for nuclear recoils in scintillators: (a) O, $\mathrm{Cd}$ and $\mathrm{W}$ ions in $\mathrm{CdWO}_{4}$; (b) $\mathrm{O}, \mathrm{W}$ and $\mathrm{Pb}$ ions in $\mathrm{PbWO}_{4}$; (c) $\mathrm{F}$ and Ce ions in $\mathrm{CeF}_{3}$; (d) $\mathrm{O}, \mathrm{Ge}$ and $\mathrm{Bi}$ ions in $\mathrm{Bi}_{4} \mathrm{Ge}_{3} \mathrm{O}_{12}$; (e) $\mathrm{Li}$ and $\mathrm{F}$ ions in $\mathrm{LiF}$; (f) $\mathrm{Zn}$ and $\mathrm{Se}$ ions in $\mathrm{ZnSe}$. 
(3) Quenching factors for $\mathrm{F}$ and Ce nuclear recoils in $\mathrm{CeF}_{3}$ scintillator (Fig. 19c) are calculated supposing $k B=11.1 \times 10^{-3} \mathrm{~g} \mathrm{MeV}^{-1} \mathrm{~cm}^{-2}$ obtained by fitting data of [58] for $\alpha$ particles (see Fig. 15).

(4) For $\mathrm{Bi}_{4} \mathrm{Ge}_{3} \mathrm{O}_{12}$ crystal scintillator $\left(\rho=7.13 \mathrm{~g} \mathrm{~cm}^{-3}\right)$, not only quenching factors for nuclear recoils were not measured, but also quenching for $\alpha$ particles was not studied in detail. $Q_{\alpha}$ values were quoted in several works, but mainly for $\simeq 5.5 \mathrm{MeV} \alpha$ particles from ${ }^{241} \mathrm{Am}$ source. The results for $E_{\alpha}=5.5 \mathrm{MeV}$ are quite different: $Q_{\alpha}=0.17$ at $20 \mathrm{mK}$ temperature [70], $Q_{\alpha}=0.20-0.21$ at a room temperature [26], and $Q_{\alpha}=0.45$ at $12 \mathrm{mK}$ temperature [71]. Result of $Q_{\alpha}=0.30 \pm 0.03$ for $E_{\alpha}=5.5-8.8 \mathrm{MeV}[72]$ is also known.

We give in Fig. 19d calculations of quenching factors for O, Ge and Bi recoils in $\mathrm{Bi}_{4} \mathrm{Ge}_{3} \mathrm{O}_{12}$ scintillator for two extreme $k B$ values: $k B=2.7 \times 10^{-3} \mathrm{~g} \mathrm{MeV}^{-1}$ $\mathrm{cm}^{-2}$ (which reproduces value of $Q_{\alpha}=0.45$ for $5.5 \mathrm{MeV} \alpha$ particle of [71]) and $k B=10.9 \times 10^{-3} \mathrm{~g} \mathrm{MeV}^{-1} \mathrm{~cm}^{-2}$ (which reproduces $Q_{\alpha}=0.17$ for 5.5 $\mathrm{MeV} \alpha$ particle [70]).

(5) Information on quenching in LiF crystal scintillator $\left(\rho=2.64 \mathrm{~g} \mathrm{~cm}^{-3}\right)$ is extremely scarce. We were able to find only one paper where value of $Q_{\alpha}=0.29$ was measured for $5.5 \mathrm{MeV} \alpha$ particles [70]. This value can be reproduced by Eq. (5) with the Birks factor $k B=2.5 \times 10^{-3} \mathrm{~g} \mathrm{MeV}^{-1} \mathrm{~cm}^{-2}$. Quenching factors for $\mathrm{Li}$ and $\mathrm{F}$ ions in $\mathrm{LiF}$ with this $k B$ are shown in Fig. 19e.

(6) ZnSe crystal scintillator (pure and doped by various elements; $\rho=5.65$ $\mathrm{g} \mathrm{cm}^{-3}$ ) is very interesting material in which an extremely low quenching is observed for $\alpha$ particles: measured values of $Q_{\alpha}$ are close to 1. For example, $Q_{\alpha}=1.0 \pm 0.1$ for $5.5 \mathrm{MeV} \alpha$ particles from ${ }^{241} \mathrm{Am}$ was obtained in [73].

Several samples of ZnSe doped by O, Al, Cd, Te were investigated in Ref. [74]. $Q_{\alpha}$ values for $\alpha$ particles of $\simeq 5.2 \mathrm{MeV}\left({ }^{239} \mathrm{Pu}\right)$ were different for crystals with different dopants: for time of a signal collection $\Delta t=12.8 \mu \mathrm{s}$, value of $Q_{\alpha}=$ 0.70 was obtained for $\mathrm{ZnSe}(\mathrm{Cd})$, and $Q_{\alpha}=0.82$ was obtained for $\mathrm{ZnSe}(\mathrm{O})$. For $\mathrm{ZnSe}(\mathrm{Te})$, which is a slow scintillator (with decay time for different components of scintillating signal as $30-80 \mu \mathrm{s}$ [74]), even values of $Q_{\alpha}>1$ were obtained $\left(Q_{\alpha}=1.25\right.$ with $\Delta t=0.6 \mu \mathrm{s}$, and 1.13 with $\left.\Delta t=12.8 \mu \mathrm{s}\right)$. Evidently even collection time of $\Delta t=12.8 \mu \mathrm{s}$ is not long enough to collect total signal in this slow scintillator. We could suppose that this is a reason of values $Q_{\alpha}>1$, and collection of a signal during longer times would give an usual situation with $Q_{\alpha}<117$.

At bolometric temperatures, when signals in ZnSe are extremely long $(>150$

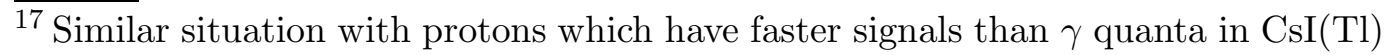
was already mentioned in section 2: $Q_{p}>1$ was obtained for $\Delta t=1 \mu \mathrm{s}$, and $Q_{p}<1$ for $\Delta t=7 \mu \mathrm{s}$. 
ms), it was possible even to obtain values of $Q_{\alpha} \simeq 4$ [75].

We have to note here that Eq. (5) supposes quenching of a signal from highly ionizing particle in comparison with that from electrons, and it is impossible to describe any enhancement on its basis (staying with physical values of $k B>0$ ). For $k B=0$, Eq. (5) gives quenching factor $Q_{i}=1$ for any particle. In Fig. 19f, we suppose usual situation when a signal is totally collected and $Q_{i}<1$. Quenching factors are drawn for Zn and Se ions in ZnSe with $k B$ value as: $k B=1.9 \times 10^{-5} \mathrm{~g} \mathrm{MeV}^{-1} \mathrm{~cm}^{-2}$ (which gives $Q_{\alpha}=0.99$ for $5.5 \mathrm{MeV}$ $\alpha$ particles) and $k B=8.4 \times 10^{-4} \mathrm{~g} \mathrm{MeV}^{-1} \mathrm{~cm}^{-2}\left(Q_{\alpha}=0.70\right.$ for $5.5 \mathrm{MeV} \alpha$ particles).

\section{Conclusions}

Semi-empirical and quite simple in realization method of calculation of quenching factors for scintillators was described in this work. It is based on the classical Birks formula with the total stopping powers for electrons and ions, and has only one parameter: the Birks factor $k B$. Value of this factor for a given scintillating material can be different in different conditions of measurements and data treatment. However, if experimental conditions and treatment of data are fixed, hypothesis that $k B$ has the same value for particles of different kinds gives reliable results. Once the $k B$ is found by fitting quenching factors for particles of one kind and in some range of energies (e.g. for $\alpha$ particles from internal contamination of a detector by $\mathrm{U} / \mathrm{Th}$ chains and/or by ${ }^{147} \mathrm{Sm},{ }^{190} \mathrm{Pt}$ with energies of a few $\mathrm{MeV}$ ), it can be used to calculate quenching factors for particles of another kinds and for another energies of interest (e.g. for low energy nuclear recoils). Many examples were given for materials which, furthermore, have different mechanisms of scintillation: organic scintillators (solid $\mathrm{C}_{8} \mathrm{H}_{8}$, and liquid $\mathrm{C}_{16} \mathrm{H}_{18}, \mathrm{C}_{9} \mathrm{H}_{12}$ ); crystal scintillators (pure $\mathrm{CdWO}_{4}, \mathrm{PbWO}_{4}$, $\mathrm{ZnWO}_{4}, \mathrm{CaWO}_{4}, \mathrm{CeF}_{3}$, and doped $\mathrm{CaF}_{2}(\mathrm{Eu}), \mathrm{CsI}(\mathrm{Tl}), \mathrm{CsI}(\mathrm{Na}), \mathrm{NaI}(\mathrm{Tl})$ ); and liquid noble gases (LXe). It was demonstrated for many cases that the method allows not only to describe measured data for ions of one kind in a reliable way but also to predict behaviour of quenching factors for other particles which sometimes is immediately confirmed by already existing experimental data - sometimes worse, sometimes better, and sometimes very good, but at least in a rough agreement. Some predictions (e.g. for LNe, LiF and others) could be checked in near future.

Stopping powers for electrons and ions are calculated with the ESTAR and SRIM codes, respectively, which in fact present to-date state-of-art software in this field. It is easy to use these programs and they are publicly available; this makes $Q_{i}$ calculations quite simple. 
Calculations with the SRIM package have some tendency to overestimate quenching factors for $\alpha$ particles at energies around $\simeq 2 \mathrm{MeV}$ and underestimate them at high energies $(>8 \mathrm{MeV})$ as can be seen in Fig. 4a for $\mathrm{CdWO}_{4}$, Fig. 5a for $\mathrm{CaF}_{2}(\mathrm{Eu})$, Fig. 8 for $\mathrm{CaWO}_{4}$, and Fig. 15 for $\mathrm{CeF}_{3}$. At the same time, calculation of the stopping powers for $\alpha$ particles with the ASTAR package gave better description of $Q_{\alpha}$ in $\mathrm{CaF}_{2}(\mathrm{Eu})$ scintillator at lower energies (see Fig. 5a). For some other materials difference between ASTAR and SRIM calculations was not big (see Fig. 2 for $\mathrm{CsI}(\mathrm{Tl})$ and Fig. 3 a for $\mathrm{C}_{8} \mathrm{H}_{8}$ ). Evidently $Q_{i}$ values will depend on how one calculates stopping powers for ions and electrons, and it is a pity that stopping powers could not be computed in framework of the same package for any particle (SRIM calculates SP for ions in any substance but does not calculate SP for electrons; and STAR gives SP for electrons in any material but SP for ions are possible only for protons and $\alpha$ particles and for a limited list of materials).

Quenching factors calculated in the presented approach in general increase at low energies, and this encourages experimental searches for dark matter particles. Estimations of quenching factors for nuclear recoils are given for some scintillators where experimental data are absent $\left(\mathrm{CdWO}_{4}, \mathrm{PbWO}_{4}\right.$, $\left.\mathrm{CeF}_{3}, \mathrm{Bi}_{4} \mathrm{Ge}_{3} \mathrm{O}_{12}, \mathrm{LiF}, \mathrm{ZnSe}\right)$.

\section{Acknowledgments}

Author is grateful to R. Bernabei, F.A. Danevich and S.K. Kim for valuable discussions, and to anonymous referee for useful suggestions. Work was supported in part by the Brain Pool program of the Korean Federation of Science and Technology Societies, and by the Project "Kosmomikrofizyka" (Astroparticle Physics) of the National Academy of Sciences of Ukraine.

\section{References}

[1] G. Bertone, D. Hooper, J. Silk, Phys. Rept. 405 (2005) 279.

[2] S.D. Steffen, Eur. Phys. J. C 59 (2009) 557.

[3] N.J.C. Spooner, J. Phys. Soc. Japan 76 (2007) 111016.

[4] R. Bernabei et al., Eur. Phys. J. C 56 (2008) 333.

[5] J.B. Birks, The Theory and Practice of Scintillation Counting, Pergamon Press, Oxford, 1964.

[6] I. Bavykina et al., IEEE Trans. Nucl. Sci. 55 (2008) 1449. 
[7] A. Calleja et al., J. Low Temp. Phys. 151 (2008) 848.

[8] H. Chagani et al., JINST 3 (2008) P06003.

[9] N. Coron et al., J. Low Temp. Phys. 151 (2008) 865.

[10] M. Kobayashi et al., Nucl. Instrum. Meth. A 592 (2008) 369.

[11] J.A. Nikkel et al., Astropart. Phys. 29 (2008) 161.

[12] P. Sorensen et al., Nucl. Instrum. Meth. A 601 (2009) 339.

[13] F.A. Danevich et al., Phys. Rev. C 67 (2003) 014310.

[14] Yu.G. Zdesenko et al., Nucl. Instrum. Meth. A 538 (2005) 657.

[15] P. Belli et al., Nucl. Phys. A 789 (2007) 15.

[16] J.B. Birks, Proc. Phys. Soc. A 64 (1951) 874.

[17] R.B. Murray, A. Meyer, Phys. Rev. 122 (1961) 815.

[18] J. Lindhard et al., Mat. Fys. Medd. K. Dan. Vidensk. Selsk. 33 (1963) 1.

[19] A. Hitachi, Astropart. Phys. 24 (2005) 247;

A. Hitachi, Proc. Int. Workshop IDM'2006, 11-16 Sept. 2006, Rhodes, Greece, p. 344;

A. Hitachi, J. Phys.: Conf. Ser. 65 (2007) 012013.

[20] D.-M. Mei, Z.-B. Yin, L.C. Stonehill, A. Hime, Astropart. Phys. 30 (2008) 12.

[21] M.J. Berger, J.S. Coursey, M.A. Zucker, J. Chang, Stopping-Power and Range Tables for Electrons, Protons, and Helium Ions, version 1.2, http://physics.nist.gov/PhysRefData/Star/Text/contents.html.

[22] J.F. Ziegler, J.P. Biersack, M.D. Ziegler, SRIM. The Stopping and Range of Ions in Matter, SRIM Co., 2008;

SRIM version 2008.04, http://www.srim.org.

[23] L. Bardelli et al., Nucl. Instrum. Meth. A 584 (2008) 129.

[24] A.N. Annenkov et al., Nucl. Instrum. Meth. A 584 (2008) 334.

[25] T. Yanagida et al., Proc. IEEE Nucl. Sci. Symp. 2007, p. 1347.

[26] E.V. Sysoeva, V.A. Tarasov, O.V. Zelenskaya, V.A. Sulyga, Nucl. Instrum. Meth. A 414 (1998) 274.

[27] R. Gwin, R.B. Murray, Phys. Rev. 131 (1963) 501.

[28] J.W. Marsh, D.J. Thomas, M. Burke, Nucl. Instrum. Meth. A 366 (1995) 340.

[29] R. Bernabei et al., Eur. Phys. J. C 53 (2008) 205.

[30] Thanks to anonymous referee for this note. 
[31] M. Bongrand (on behalf of the SuperNEMO collaboration), AIP Conf. Proc. 897 (2007) 14.

[32] H.O. Back et al., Nucl. Instrum. Meth. A 584 (2008) 98.

[33] J. Hong et al., Astropart. Phys. 16 (2002) 333.

[34] T. Fazzini et al., Nucl. Instrum. Met. A 410 (1998) 213.

[35] R.B. Firestone et al., Table of isotopes, 8-th ed., John Wiley, New York, 1996 and CD update, 1998.

[36] R. Hazama et al., Nucl. Instrum. Met. A 482 (2002) 297.

[37] S. Umehara et al., Phys. Rev. C 78 (2008) 058501.

[38] F.A. Danevich et al., Nucl. Instrum. Meth. A 556 (2006) 259.

[39] H. Kraus et al., Nucl. Instrum. Meth. A 600 (2009) 594.

[40] F.A. Danevich et al., Nucl. Instrum. Meth. A 544 (2005) 553.

[41] G. Angloher et al., Astropart. Phys. 23 (2005) 325.

[42] Th. Jagemann et al., Astropart. Phys. 26 (2006) 269.

[43] J. Ninkovic et al., Nucl. Instrum. Meth. A 564 (2006) 567.

[44] I. Bavykina et al., Astropart. Phys. 28 (2007) 489.

[45] S. Pecourt et al., Astropart. Phys. 11 (1999) 457.

[46] H. Park et al., Nucl. Instrum. Meth. A 491 (2002) 460.

[47] M.Z. Wang et al., Phys. Lett. B 536 (2002) 203.

[48] T.Y. Kim et al., Nucl. Instrum. Meth. A 500 (2003) 337.

[49] Y.F. Zhu et al., Nucl. Instrum. Meth. A 557 (2006) 490.

[50] D.R. Tovey et al., Phys. Lett. B 433 (1998) 150.

[51] E. der Mateosian, M. McKeown, C.O. Muehlhause, Phys. Rev. 101 (1956) 967;

J.C. Barton, Appl. Radiat. Isot. 47 (1996) 997.

[52] N.J.C. Spooner et al., Phys. Lett. B 321 (1994) 156.

[53] G. Gerbier et al., Astropart. Phys. 11 (1999) 287.

[54] E. Simon et al., Nucl. Instrum. Meth. A 507 (2003) 643.

[55] K. Fushimi et al., Phys. Rev. C 47 (1993) 425.

[56] R. Bernabei et al., Phys. Lett. B 389 (1996) 757.

[57] R. Bernabei et al., Nucl. Instrum. Meth. A 592 (2008) 297.

[58] P. Belli et al., Nucl. Instrum. Meth. A 498 (2003) 352. 
[59] D.R. Lide (ed.), CRC Handbook of Chemistry and Physics, 84-th ed., CRC Press, 2003.

[60] R. Bernabei et al., Phys. Lett. B 436 (1998) 379.

[61] V. Chepel et al., Astropart. Phys. 26 (2006) 58.

[62] R. Bernabei et al., Eur. Phys. J. direct C 11 (2001) 1.

[63] M. Tanaka et al., Nucl. Instrum. Meth. A 457 (2001) 454.

[64] E. Aprile et al., Phys. Rev. D 79 (2009) 045807.

[65] F. Arneodo et al., Nucl. Instrum. Meth. A 449 (2000) 147.

[66] D. Akimov et al., Phys. Lett. B 524 (2002) 245.

[67] R. Brunetti et al., New Astron. Rev. 49 (2005) 265;

R. Brunetti et al., WARP Proposal, http://warp.lngs.infn.it.

[68] A. Hitachi et al., Phys. Rev. A 35 (1987) 3956.

[69] P. Peiffer et al., JINST 3 (2008) P08007.

[70] N. Coron et al., Nucl. Instrum. Meth. A 520 (2004) 159.

[71] P. Meunier et al., Appl. Phys. Lett. 75 (1999) 1335.

[72] Z. Dlouhy et al., Nucl. Instrum. Meth. A 317 (1992) 604.

[73] F.A. Danevich et al., Instr. Exp. R. 32 (1989) 1059.

[74] W.G. Lee et al., J. Korean Phys. Soc. 48 (2006) 47.

[75] S. Pirro, talk at the 6th ILIAS Ann. Meeting, Dresden, Germany, 16-19 February 2009; http://www-ilias.cea.fr. 